\title{
Link Floer homology detects the Thurston norm
}

\author{
YI NI
}

\begin{abstract}
We prove that, for a link $L$ in a rational homology 3-sphere, the link Floer homology detects the Thurston norm of its complement. This result has been proved by Ozsváth and Szabó for links in $S^{3}$. As an ingredient of the proof, we show that knot Floer homology detects the genus of null-homologous links in rational homology spheres, which is a generalization of an earlier result of the author. Our argument uses the techniques due to Ozsváth and Szabó, Hedden and the author.
\end{abstract}

57R58, 57M27; 57R30

\section{Introduction}

Link Floer homology was introduced by Ozsváth and Szabó [13], as a multifiltered theory for links in rational homology 3 -spheres. This theory generalizes an earlier invariant for knots, the knot Floer homology (see Ozsváth and Szabó [11] and Rasmussen [15]). One interesting topic in Floer theory is the relationship with the Thurston norm. For knot (and link) Floer homology, this topic was studied for links in integer homology 3-spheres by Ozsváth and Szabó [10;14] and the author [8]. In particular, Ozsváth and Szabó showed that, for a link $L \subset S^{3}$, the link Floer homology detects the Thurston norm of the complement of $L$. Although not stated explicitly, their proof actually works for links in integer homology spheres.

In the current paper, we will generalize Ozsváth and Szabó's result to links in rational homology 3-spheres.

In Section 4.4, we will define an affine function

$$
\mathfrak{H}: \underline{\operatorname{Spin}^{c}}(Y, L) \rightarrow H^{2}(Y, L ; \mathbb{Q}) .
$$

Then the link Floer homology provides a function

$$
y(h)=\max _{\left\{\mathfrak{r} \in \underline{\operatorname{Spin}}^{c}(Y, L) \mid \widehat{H F L}(Y, L, \mathfrak{r}) \neq 0\right\}}\langle\mathcal{H}(\mathfrak{r}), h\rangle .
$$

defined by 
Theorem 1.1 Suppose $L$ is an oriented link in a rational homology 3-sphere $Y$ and $M=Y-\operatorname{int}(\operatorname{Nd}(L))$. Suppose $h \in H_{2}(M, \partial M ; \mathbb{Q})$ is an integral class. Then $h$ can be represented by a properly embedded surface without sphere components. Let $\chi(h)$ be the maximal possible value of the Euler characteristic of such surfaces. Then

$$
-\chi(h)+\sum_{i=1}^{l}\left|h \cdot\left[\mu_{i}\right]\right|=2 y(h) .
$$

Here $\mu_{1}, \ldots, \mu_{l}$ are the meridians of the components of $L$.

Remark 1.2 The term $-\chi(h)$ is almost the Thurston norm of $h$ [19]. In fact, if the boundary tori of $M$ are all incompressible, then we can rewrite the equality in the above theorem as

$$
x(h)+\sum_{i=1}^{l}\left|h \cdot\left[\mu_{i}\right]\right|=2 y(h),
$$

where here $x(\cdot)$ is the Thurston norm.

Remark 1.3 Suppose $M$ is a compact 3-manifold with boundary consisting of tori, and $H_{2}(M)=0$. Then $M$ is the complement of a link in a rational homology sphere. Theorem 1.1 gives a criterion to determine whether any component of $\partial M$ is compressible, in the terms of link Floer homology. If $T$ is a torus in a rational homology 3-sphere $Y$, then $T$ splits $Y$ into two rational homology solid tori. Thus Theorem 1.1 also gives a criterion to determine whether $T$ is compressible.

Incompressible tori play a very important role in "traditional 3-dimensional topology". We hope that the above observation will be useful for studying the relationship between Floer homology and traditional 3-dimensional topology.

The paper is organized as follows. Section 2 contains a rather general result about the existence of longitudinal foliations. This result will be the starting point of our proof. Then, in Section 3, we generalize the main result in our paper [8] to null-homologous oriented links in rational homology spheres. In Section 4, we give some preliminaries on link Floer homology. In particular, we discuss the relative $\operatorname{Spin}^{c}$ structures. Section 5 will be devoted to the proof of the main theorem. We use the "cabling trick" from Ozsváth and Szabó [14], as well as the techniques from Hedden [4], to reduce the general case of our main theorem to the case proved in Section 3. 
Acknowledgements We are grateful to David Gabai and Zoltán Szabó for some helpful conversations. We wish to thank Efstratia Kalfagianni for informing us Kaiser's work [5]. We particularly thank the referee for a thorough reading of an earlier version of this paper, for many constructive suggestions and for pointing out a serious mistake.

The first version of this paper was carried out when the author was a graduate student at Princeton University. The author was partially supported by a Graduate School Centennial fellowship at Princeton University during the course of this work.

\section{Longitudinal foliations}

As in [10] and [8], when one tries to relate Floer homology with Thurston norm, the first step is always to establish an existence result about taut foliations. In this section, we are going to establish the corresponding result we need.

Definition 2.1 $M$ is an $n$-dimensional manifold. A smooth (codimension-1) foliation $\mathscr{F}$ of $M$ is a smooth integrable hyperplane field on $M$. A leaf $L$ of $\mathscr{F}$ is a maximal path-connected integral submanifold for $\mathscr{F}$.

By abuse of notation, we also denote the collection of the leaves by $\mathscr{F}$.

From now on, we assume the foliation is co-oriented, namely, there exists a unit vector field on the manifold, which is transverse to the foliation everywhere.

If $\gamma$ is a path in a leaf $L$, then $\mathscr{F}$ defines a parallel transport in a small neighborhood of $\gamma$. Let $a, b$ be the two ends of $\gamma$, there are two small transversals $I_{a}, I_{b}$ passing through $a, b$, so that the parallel transport along $\gamma$ defined by $\mathscr{F}$ gives a diffeomorphism of $I_{a}$ onto $I_{b}$. Moreover, if $\gamma$ is a loop with base point $b$, then the germ of the diffeomorphism at $b$ is called the holonomy along the loop $\gamma$.

Every closed orientable 3-manifold admits a smooth foliation [16, Theorem 10.A.15]. So in order to extract useful information about 3-manifolds out of foliations, one needs some further restriction on the foliations.

Definition 2.2 Let $\mathscr{F}$ be a foliation of a 3-manifold $M$. $\mathscr{F}$ is taut if there exists a closed curve intersecting every leaf of $\mathscr{F}$ transversely.

In order to study knot Floer homology, one always needs some additional conditions on the taut foliations. For example, the foliations should be "longitudinal". The definition is as follows. 
Definition 2.3 Suppose $K \subset Y$ is a null-homologous knot and $\mathscr{F}$ is a taut foliation of $Y-\operatorname{int}(\operatorname{Nd}(K))$. We say that $\mathscr{F}_{F}$ is a longitudinal foliation, if the restriction of $\mathscr{F}_{F}$ on $\partial \mathrm{Nd}(K)$ consists of longitudes.

Gabai shows that longitudinal foliations exist in many cases, including the classical knots [3]. In fact, we are going to prove the following rather general result about the existence of longitudinal foliations.

Proposition 2.4 Suppose $K \subset Y$ is a null-homologous knot, $Y-K$ is irreducible. Then for any fibred knot $J \subset S^{3}$ with sufficiently large genus, $Y-\operatorname{int}(\operatorname{Nd}(K \# J))$ admits a smooth longitudinal foliation with a compact leaf, which is a minimal genus Seifert surface of $K \# J$.

Proposition 2.4 is a weak form of the following theorem due to Gabai.

Theorem (Gabai [1]) Suppose $K_{i} \subset Y_{i}$ are nontrivial null-homologous knots, $Y_{i}-$ $K_{i}$ are irreducible, $i=1,2$. Then $Y_{1} \# Y_{2}-\operatorname{int}\left(\mathrm{Nd}\left(K_{1} \# K_{2}\right)\right)$ admits a smooth longitudinal foliation with a compact leaf, which is a minimal genus Seifert surface of $K_{1} \# K_{2}$.

Proof of Proposition 2.4 Suppose $F \subset M=Y-\operatorname{int}(\operatorname{Nd}(K))$ is a minimal genus Seifert surface for $K$. By the main theorem in [2], there exists a taut smooth foliation F of $M$, so that

(1) $\mathscr{F} \pitchfork \partial M$, and $\mathscr{F} \mid \partial M$ has no Reeb component,

(2) $F$ is a leaf of $\mathscr{F}$,

(3) if $\theta$ is a closed curve in $F, f:(-\varepsilon, \varepsilon) \rightarrow(-\delta, \delta)$ is a representative of the germ of the holonomy along $\theta$, then

$$
\frac{\mathrm{d}^{k} f}{\mathrm{~d} t^{k}}(0)= \begin{cases}1, & k=1, \\ 0, & k>1\end{cases}
$$

Here (3) holds by the Induction Hypothesis (iii) in the proof of [2, Theorem 5.1].

Cut $M$ open along $F$, we get a sutured manifold $\left(M_{0}, \gamma_{0}\right)$, and $\mathscr{F}$ becomes a foliation $\mathscr{F}_{0}$ of $M_{0}$. The suture $\gamma_{0}$ is an annulus. By the above condition (1), $\mathscr{F}_{0} \mid \gamma_{0}$ is determined by a global holonomy $f: I \rightarrow I$. Namely, pick the square $I \times I$, foliated by $I \times t$ 's. Glue $0 \times I$ with $1 \times I$ by a diffeomorphism $f$, then the induced foliation on $S^{1} \times I$ is equivalent to the foliation $\mathscr{F}_{0} \mid \gamma_{0}$. We can view $\gamma_{0}$ as the union of two 
squares $a \times I$ and $b \times I$, so that the restriction of the foliation in $a \times I$ consists of $a \times t$ 's, and the holonomy takes place in $b \times I$.

Suppose $D_{8}$ is an octagon with edges $a_{1}, b_{1}, a_{2}, b_{2}, \ldots, a_{4}, b_{4}$ in cyclic order. Consider $D_{8} \times I$, foliated by $D_{8} \times t$ 's. Let $g, h: I \rightarrow I$ be two diffeomorphisms with the two ends fixed. We glue $b_{1} \times I$ with $b_{3} \times I$ by the map $-\mathrm{id} \times g$, glue $b_{2} \times I$ with $b_{4} \times I$ by the map $-\mathrm{id} \times h$. The new manifold is $R \times I$, with an induced foliation $\mathscr{G}$. Here $R$ is a genus- 1 compact surface with one boundary component. Obviously, $\mathscr{G} \mid \partial R \times I$ has a global holonomy $[g, h]$. We can view $\partial R \times I$ as the union of two squares $a^{\prime} \times I$ and $b^{\prime} \times I$, so that the restriction of the foliation in $a^{\prime} \times I$ consists of $a^{\prime} \times t^{\prime}$ s, and the holonomy takes place in $b^{\prime} \times I$.

Now we glue the two sutured manifolds $\left(M_{0}, \gamma_{0}\right)$ and $(R \times I, \partial R \times I)$ together, so that $a \times I$ is glued to $a^{\prime} \times I$ by the identity. Then the new sutured manifold $\left(M_{1}, \gamma_{1}\right)$ has an induced foliation $\mathscr{F}_{1}$, so that $\mathscr{F}_{1} \mid \gamma_{1}$ has a global holonomy $f \circ[g, h]$.

Repeat the above construction $m$ times, we get a foliated sutured manifold $\left(M_{m}, \gamma_{m}\right)$, which is the union of $\left(M_{0}, \gamma_{0}\right)$ and $\left(R_{m} \times I, \partial R_{m} \times I\right)$ along a square in the suture, and the holonomy of the foliation on $\gamma_{m}$ is

$$
f \circ\left[g_{1}, h_{1}\right] \circ\left[g_{2}, h_{2}\right] \circ \cdots \circ\left[g_{m}, h_{m}\right] .
$$

Here $R_{m}$ is a compact genus- $m$ surface with one boundary component. Denote this foliation of $M_{m}$ by $\mathscr{F}_{m}$. Now we can make use of the following theorem.

Theorem (Mather-Sergeraert-Thurston [18]; see also Gabai [2]) If $f: I \rightarrow I$ is a $C^{\infty}$ map satisfying

$$
\frac{\mathrm{d}^{k} f}{\mathrm{~d} t^{k}}(\alpha)= \begin{cases}1, & k=1 \\ 0, & k>1\end{cases}
$$

for $\alpha \in\{0,1\}$, then there exist $C^{\infty}$ diffeomorphisms $g_{i}, h_{i}: I \rightarrow I, i=1, \ldots, n$, satisfying the above conditions so that

$$
f \circ\left[g_{1}, h_{1}\right] \circ\left[g_{2}, h_{2}\right] \circ \cdots \circ\left[g_{n}, h_{n}\right]=\mathrm{id}
$$

Hence when $m \geq n$, one can choose the holonomies $g_{i}, h_{i}, i=1, \ldots, m$, so that the holonomy of $\mathscr{F}_{m} \mid \gamma_{m}$ is the identity, thus $\mathscr{F}_{m} \mid \gamma_{m}$ consists of closed curves.

Suppose $J \subset S^{3}$ is a fibred knot with genus $m, G$ is a minimal genus Seifert surface of $J$. Consider the knot $K \# J$, with Seifert surface $F^{\prime}$, which is the boundary connected sum of $F$ and $G$. If we cut $Y-\operatorname{int}(\operatorname{Nd}(K \# J))$ open along $F^{\prime}$, the sutured manifold we get is just $\left(M_{m}, \gamma_{m}\right)$. Let $F_{+}^{\prime}, F_{-}^{\prime}$ be the two copies of $F^{\prime}$ in $\partial M_{m}$. 
Starting with the compact manifold $M_{m}$, we can glue $F_{+}^{\prime}$ and $F_{-}^{\prime}$ together to get the knot exterior $Y-\operatorname{int}(\operatorname{Nd}(K \# J))$. The above foliation $\mathscr{F}_{m}$ then becomes a smooth longitudinal foliation of $Y-\operatorname{int}(\operatorname{Nd}(K \# J))$, such that $F^{\prime}$ is a compact leaf.

\section{Genera of links in rational homology spheres}

In this section, we are going to follow the procedure in [8] to generalize the main result there to null-homologous links in rational homology 3-spheres.

Theorem 3.1 Suppose $L$ is a null-homologous oriented link in a closed 3-manifold $Z, H_{1}(Z ; \mathbb{Q})=0 .|L|$ denotes the number of components of $L$, and $\chi(L)$ denotes the maximal Euler characteristic of the Seifert surfaces bounded by $L$. Then

$$
\frac{|L|-\chi(L)}{2}=\max \{i \mid \widehat{H F K}(Z, L, i) \neq 0\} .
$$

Let $L$ be a null-homologous oriented $l$-component link in a rational homology 3sphere $Z$. Ozsváth and Szabó define a knot $\kappa(L) \subset \kappa(Z)$, where here $\kappa(Z) \cong$ $Z \#(l-1) S^{1} \times S^{2}$ is obtained by adding $l-13$-dimensional tubes $R_{1}, \ldots, R_{l-1}$ to $Z$. Suppose $P_{i}$ is the belt sphere of the tube $R_{i}$. The knot $\kappa(L)$ intersects $P_{i}$ in exactly 2 points, we can remove two disks from $P_{i}$ at these two points, then glue in a long and thin (2-dimensional) tube along an arc in $\kappa(L)$, so as to get a torus $T_{i}$. $T_{i}$ is homologous to $P_{i}$, but disjoint from $\kappa(L)$. These tori generate $H_{2}(\kappa(Z)-\kappa(L) ; \mathbb{Z})$. Let $(Y, K)=(\kappa(Z), \kappa(L))$. Let $G$ be a minimal genus Seifert surface of $K$, and $Y_{0}$ be the manifold obtained by 0 -surgery on $K$. By [8, Remark 3.2], we can assume $G$ is obtained by adding $l-1$ bands to a Seifert surface $F$ of $L$ with maximal Euler characteristic. Hence $\chi(G)=\chi(F)-(l-1)$. Let $\widehat{G}$ be the extension of $G$ in $Y_{0}$ obtained by gluing a disk to $G$.

Proposition 3.2 Let $L$ be a null-homologous oriented link in a rational homology 3-sphere $Z$, with irreducible complement. After doing connected sum with some fibred knots in $S^{3}$, we get a new link $L^{*}$. We consider $\left(Y^{*}, K^{*}\right)=\left(\kappa(Z), \kappa\left(L^{*}\right)\right)$, and the 0 -surgered space $Y_{0}^{*}$. The conclusion is: for a suitably chosen $L^{*}, Y_{0}^{*}$ can be embedded into a closed symplectic 4-manifold $(X, \Omega)$, so that $X=X_{1} \cup_{Y_{0}^{*}} X_{2}$, $b_{2}^{+}\left(X_{j}\right)>0$, and

$$
\int_{T_{i}^{*}} \Omega=0
$$

for all $i$. Moreover,

$$
\left\langle c_{1}(\mathfrak{k}(\Omega)),\left[\widehat{G^{*}}\right]\right\rangle=2-2 g\left(\widehat{G^{*}}\right) .
$$


Having Proposition 2.4, the proof of Proposition 3.2 is the same as the proof of [8, Proposition 3.12]. So we just omit it here.

We also state the following lemma without giving the proof, since its proof is not different from the proof of [8, Lemma 4.1].

Lemma 3.3 $\left(Y^{*}, K^{*}\right)$ is as before. Let $d$ be an integer satisfying

$$
\widehat{H F K}\left(Y^{*}, K^{*}, i\right)=0 \quad \text { for } i \geq d,
$$

and suppose that $d>1$. Then

$$
H F^{+}\left(Y_{0}^{*},[d-1]\right)=0,
$$

where

$$
H F^{+}\left(Y_{0}^{*},[d-1]\right)=\underset{\left\langle c_{1}(\mathfrak{s}),\left[\widehat{G}^{*}\right]\right\rangle=2(d-1)}{\bigoplus} H F^{+}\left(Y_{0}^{*}, \mathfrak{s}\right)
$$

Proof of Theorem 3.1 (Compare the proof of [8, Theorem 1.1].) Suppose $L_{1}, L_{2}$ are null-homologous oriented links in $Z_{1}, Z_{2}$, respectively. We have

$$
\begin{aligned}
\widehat{H F K}\left(Z_{1}, L_{1}\right) \otimes \widehat{H F}\left(Z_{2}\right) & \cong \widehat{H F K}\left(Z_{1} \# Z_{2}, L_{1}\right), \\
\widehat{H F K}\left(Z_{1} \# Z_{2}, L_{1} \# L_{2}\right) \otimes \widehat{H F}\left(S^{2} \times S^{1}\right) & \cong \widehat{H F K}\left(Z_{1} \# Z_{2}, L_{1} \sqcup L_{2}\right) .
\end{aligned}
$$

By the above formulas, we can assume $Z-L$ is irreducible. Now apply Proposition 3.2 to get a symplectic 4-manifold $(X, \Omega), X=X_{1} \cup_{Y_{0}^{*}} X_{2}$, with $b_{2}^{+}\left(X_{j}\right)>0$, $\int_{T_{i}^{*}} \Omega=0$, and

$$
\left\langle c_{1}(\mathfrak{k}(\Omega)),\left[\widehat{G^{*}}\right]\right\rangle=\chi\left(\widehat{G^{*}}\right)<0 .
$$

Let $\delta: H^{1}\left(Y_{0}^{*}\right) \rightarrow H^{2}(X)$ be the connecting homomorphism in the Mayer-Vietoris sequence for the decomposition of $X$ into $X_{1}$ and $X_{2}$. The sum

$$
\sum_{\eta \in H^{1}\left(Y_{0}^{*}\right)} \Phi_{X, \mathfrak{k}(\Omega)+\delta \eta}
$$

is calculated by a homomorphism which factors through $H F^{+}\left(Y_{0}^{*},\left.\mathfrak{k}(\Omega)\right|_{Y_{0}^{*}}\right)$.

The cohomology group $H^{1}\left(Y_{0}^{*}\right) \cong \mathbb{Z}^{l}$ is generated by the Poincare duals of $\left[T_{1}^{*}\right]$, $\left[T_{2}^{*}\right], \ldots,\left[T_{l-1}^{*}\right]$ and $\left[\widehat{G^{*}}\right]$. So the $\operatorname{Spin}^{c}$ structures in (1) are precisely

$$
\mathfrak{k}(\Omega)+\sum_{i=1}^{l-1} a_{i} \operatorname{PD}\left(\left[T_{i}^{*}\right]\right)+b \operatorname{PD}\left(\left[\widehat{G^{*}}\right]\right) \quad\left(a_{i}, b \in \mathbb{Z}\right) .
$$


Here PD is the Poincare duality map in $X$. The first Chern classes of these $\operatorname{Spin}^{c}$ structures are

$$
c_{1}(\mathfrak{k}(\Omega))+2 \sum_{i=1}^{l-1} a_{i} \mathrm{PD}\left(\left[T_{i}^{*}\right]\right)+2 b \operatorname{PD}\left(\left[\widehat{G^{*}}\right]\right) .
$$

By the degree shifting formula, the degrees of the terms in (1) are

$$
\begin{aligned}
& \frac{\left(c_{1}(\mathfrak{k}(\Omega))+2 \sum a_{i} \operatorname{PD}\left(\left[T_{i}^{*}\right]\right)+2 b \operatorname{PD}\left(\left[\widehat{G}^{*}\right]\right)\right)^{2}-2 \chi(X)-3 \sigma(X)}{4} \\
= & \frac{\left(c_{1}(\mathfrak{k}(\Omega))^{2}-2 \chi(X)-3 \sigma(X)\right.}{4}+\sum a_{i}\left\langle c_{1}(\mathfrak{k}(\Omega)),\left[T_{i}^{*}\right]\right\rangle+b\left\langle c_{1}(\mathfrak{k}(\Omega)),\left[\widehat{G^{*}}\right]\right\rangle \\
= & \frac{\left(c_{1}(\mathfrak{k}(\Omega))^{2}-2 \chi(X)-3 \sigma(X)\right.}{4}+b \chi\left(\widehat{G^{*}}\right) .
\end{aligned}
$$

Since $\chi\left(\widehat{G^{*}}\right) \neq 0$, the terms which have the same degree as $\Phi_{X, \mathfrak{k}(\Omega)}$ are precisely those correspond to $\mathfrak{k}(\Omega)+\sum a_{i} \operatorname{PD}\left(\left[T_{i}^{*}\right]\right)$. By [12, Theorem 1.1] and the fact that $\int_{T_{i}^{*}} \Omega=0, \Phi_{X, \mathfrak{k}(\Omega)}$ is the only nontrivial term at this degree. So $H F^{+}\left(Y_{0}^{*},\left.\mathfrak{k}(\Omega)\right|_{Y_{0}^{*}}\right)$ is nontrivial. Now apply Lemma 3.3, we get our desired result for $L^{*}$.

The result for $L$ holds by the connected sum formula.

As a corollary, we have:

Corollary 3.4 Suppose $Z$ is a rational homology 3 -sphere, $L_{+}, L_{-}, L_{0} \subset Z$ are 3 null-homologous oriented links, which differ at a crossing as in the skein relation. Then two of the three numbers

$$
\chi\left(L_{+}\right), \chi\left(L_{-}\right), \chi\left(L_{0}\right)-1,
$$

are equal and not larger than the third.

Proof In the local picture of the skein relation, if the two strands in $L_{-}$belong to the same component, then $\left|L_{0}\right|=\left|L_{+}\right|+1$, and there is a surgery exact triangle relating $\widehat{H F K}\left(Z, L_{-}\right), \widehat{H F K}\left(Z, L_{+}\right)$and $\widehat{H F K}\left(Z, L_{0}\right)$ [11]. If $\chi\left(L_{+}\right)<\chi\left(L_{-}\right)$, then $\widehat{H F K}\left(Z, L_{-},\left(\left|L_{+}\right|-\chi\left(L_{+}\right)\right) / 2\right)=0$, hence

$$
\widehat{H F K}\left(Z, L_{+}, \frac{\left|L_{+}\right|-\chi\left(L_{+}\right)}{2}\right) \cong \widehat{H F K}\left(Z, L_{0}, \frac{\left|L_{+}\right|-\chi\left(L_{+}\right)}{2}\right) .
$$

It follows from Theorem 3.1 that

$$
\left|L_{0}\right|-\chi\left(L_{0}\right)=\left|L_{+}\right|-\chi\left(L_{+}\right),
$$


so $\chi\left(L_{+}\right)=\chi\left(L_{0}\right)-1$. Similarly, one can show that if $\chi\left(L_{-}\right)<\chi\left(L_{+}\right)$, then $\chi\left(L_{-}\right)=\chi\left(L_{0}\right)-1$, and if $\chi\left(L_{-}\right)=\chi\left(L_{+}\right)$, then $\chi\left(L_{-}\right) \leq \chi\left(L_{0}\right)-1$.

If the two strands in $L_{-}$belong to different components, then $\left|L_{0}\right|=\left|L_{-}\right|-1$, and there is an exact triangle relating $\widehat{H F K}\left(Z, L_{-}\right), \widehat{H F K}\left(Z, L_{+}\right)$and $\widehat{H F K}\left(Z, L_{0}\right) \otimes V$. Here $V=V_{-1} \oplus V_{0} \oplus V_{+1}, V_{ \pm 1} \cong \mathbb{Z}$ are supported in filtration level \pm 1 , and $V_{0} \cong \mathbb{Z} \oplus \mathbb{Z}$ is supported in filtration level 0 . An argument similar to the one in the last paragraph gives the desired result.

The above result was first proved for links in $S^{3}$ by Scharlemann and Thompson [17]. Then Kaiser proved a much more general theorem for links in irreducible rational homology 3-spheres [5]. Kalfagianni also proved Scharlemann and Thompson's result for certain links in irreducible homology 3-spheres, and applied it to study the convergence of the HOMFLY power series link invariants in [6].

\section{Preliminaries on link Floer homology}

In [13], Ozsváth and Szabó defined link Floer homology for oriented links in rational homology 3-spheres. We will briefly review the definition and some basic properties.

\subsection{Relative $\operatorname{Spin}^{c}$ structures}

Let $M$ be a compact 3-manifold with boundary consisting of tori. There is a canonical isotopy class of translation invariant vector fields on the torus. Let $v_{1}$ and $v_{2}$ be two nowhere vanishing vector fields on $M$, whose restriction on each component of $\partial M$ is the canonical translation invariant vector field. We say $v_{1}$ and $v_{2}$ are homologous, if they are homotopic in the complement of a ball in $M$, and the homotopy is through nowhere vanishing vector fields which restrict to the canonical class on $\partial M$. The homology classes of such vector fields are called relative $\operatorname{Spin}^{c}$ structures on $M$, and the set of all relative $\operatorname{Spin}^{c}$ structures is denoted by $\underline{\operatorname{Spin}^{c}}(M, \partial M) \cdot \underline{\operatorname{Spin}^{c}}(M, \partial M)$ is an affine space over $H^{2}(M, \partial M)$.

When $L$ is an oriented link in a closed oriented 3-manifold $Y$, let $M=Y-\operatorname{int}(\operatorname{Nd}(L))$. Then we also denote $\underline{\operatorname{Spin}^{c}}(M, \partial M)$ by $\underline{\operatorname{Spin}^{c}}(Y, L)$.

There is a natural involution

$$
J: \underline{\operatorname{Spin}^{c}}(M, \partial M) \rightarrow \underline{\operatorname{Spin}^{c}}(M, \partial M) .
$$

If $\mathfrak{r} \in \underline{\operatorname{Spin}}^{c}(M, \partial M)$ is represented by a vector field $v$, then $J(\mathfrak{r})$ is represented by the vector field $-v$. (Note that $-v \mid \partial M$ is still the canonical isotopy class on $\partial M$.) Given $\mathfrak{r}, \mathfrak{r}-J(\mathfrak{r})$ is an element in $H^{2}(M, \partial M)$, denoted by $c_{1}(\mathfrak{r})$. 


\subsection{Heegaard diagrams and $\operatorname{Spin}^{c}$ structures}

Suppose $L$ is an oriented link in a rational homology sphere $Y^{3},(\Sigma, \boldsymbol{\alpha}, \boldsymbol{\beta}, \mathbf{w}, \mathbf{z})$ is a (generic) balanced $2 l$-pointed Heegaard diagram associated to the pair $(Y, L)$. There is a map

$$
\underline{\mathfrak{s}}_{\mathbf{w}, \mathbf{z}}: \mathbb{T}_{\alpha} \cap \mathbb{T}_{\beta} \rightarrow \underline{\operatorname{Spin}^{c}}(Y, L),
$$

defined in [13]. We sketch the definition of $\underline{\mathfrak{s}}_{\mathbf{w}, \mathbf{z}}$ as follows.

Let $f: Y \rightarrow[0,3]$ be a Morse function corresponding to the Heegaard diagram, $\nabla f$ is the gradient vector field associated to $f$. Let $\gamma_{\mathbf{w}}$ be the union of the flowlines of $\nabla f$, such that each of these flowlines passes through a point in $\mathbf{w}$, and connects an index -0 critical point to an index -3 critical point. Similarly, define $\gamma_{\mathbf{z}}$. Suppose $\mathbf{x} \in \mathbb{T}_{\alpha} \cap \mathbb{T}_{\beta}$, then $\gamma_{\mathbf{x}}$ denotes the union of the flowlines connecting index-1 critical points to index -2 critical points, and passing through the points in $\mathbf{x}$.

We construct a nowhere vanishing vector field $v$. Make $v$ identical with $\nabla f$ outside a neighborhood $\operatorname{Nd}\left(\gamma_{\mathbf{w}} \cup \gamma_{\mathbf{z}} \cup \boldsymbol{\gamma}_{\mathbf{x}}\right)$. Then one can extend $v$ over the balls $\operatorname{Nd}\left(\boldsymbol{\gamma}_{\mathbf{x}}\right)$. We can also extend $v$ over $\operatorname{Nd}\left(\gamma_{\mathbf{w}} \cup \gamma_{\mathbf{z}}\right)$, so that the closed orbits of $v$, which pass through points in $\mathbf{w}$ and $\mathbf{z}$, give the oriented link $L=\gamma_{\mathbf{z}}-\gamma_{\mathbf{w}}$. There may be many different choices to extend $v$ over $\operatorname{Nd}\left(\gamma_{\mathbf{w}} \cup \gamma_{\mathbf{z}}\right)$, we choose the extension as in [13, Figure 2].

Now we let $\underline{\mathfrak{s}}_{\mathbf{w}, \mathbf{z}}(\mathbf{x})$ be the relative $\operatorname{Spin}^{c}$ structure given by $\left.v\right|_{Y-\mathrm{Nd}(L)}$. It is easy to check that $\underline{\mathfrak{s}}_{\mathbf{w}, \mathbf{z}}$ is a well-defined map.

\subsection{Link Floer homology}

Let $\mathbb{F}_{2}$ be the field consisting of 2 elements. For $\mathfrak{r} \in \operatorname{Spin}^{c}(Y, L), \widehat{C F L}(Y, L, \mathfrak{r})$ is a

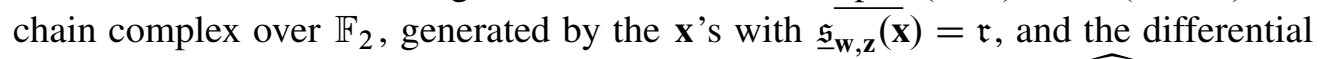
counts holomorphic disks with $n_{\mathbf{w}}(\phi)=n_{\mathbf{z}}(\phi)=0$. The homology of $\widehat{C F L}(Y, L, \mathfrak{r})$ is denoted by $\widehat{H F L}(Y, L, \mathfrak{r})$. And the link Floer homology is

$$
\widehat{H F L}(Y, L)=\bigoplus_{\mathfrak{r} \in \operatorname{Spin}^{c}(Y, L)} \widehat{H F L}(Y, L, \mathfrak{r})
$$

$\widehat{H F L}$ enjoys certain symmetries. In particular, as in [13, Proposition 8.2], we have:

Lemma 4.1 Let $L$ be an oriented link in a rational homology sphere $Y, \mu_{1}, \ldots, \mu_{l}$ denote the meridians of the components of $L$. Then

$$
\widehat{H F L}(Y, L, \mathfrak{r}) \cong \widehat{H F L}\left(Y, L, J(\mathfrak{r})+\sum_{i=1}^{l} \operatorname{PD}\left[\mu_{i}\right]\right) .
$$




\subsection{An Alexander $\mathbb{Q}^{l}$ - grading}

With the notation as above, we define a function

$$
\mathfrak{H}: \underline{\operatorname{Spin}^{c}}(Y, L) \rightarrow H^{2}(Y, L ; \mathbb{Q})
$$

as follows. Given $\mathfrak{r} \in \operatorname{Spin}^{c}(Y, L)$, let

$$
\mathfrak{H}(\mathfrak{r})=\frac{c_{1}(\mathfrak{r})-\sum_{i=1}^{l} \operatorname{PD}\left(\left[\mu_{i}\right]\right)}{2} .
$$

Moreover, if $\mathbf{x} \in \mathbb{T}_{\alpha} \cap \mathbb{T}_{\beta}$, we define

$$
\mathfrak{h}_{\mathbf{w}, \mathbf{z}}(\mathbf{x})=\mathfrak{H}\left(\underline{\mathfrak{s}}_{\mathbf{w}, \mathbf{z}}(\mathbf{x})\right) \text {. }
$$

Given $\mathbf{x}, \mathbf{y} \in \mathbb{T}_{\alpha} \cap \mathbb{T}_{\beta}$, there exist an arc $a \subset \mathbb{T}_{\alpha}$ and an arc $b \subset \mathbb{T}_{\beta}$, both connecting $\mathbf{x}$ to $\mathbf{y}$. Let $\omega(\mathbf{x}, \mathbf{y})=a-b$ be a closed curve, $\omega$ can also be viewed as a curve in $\Sigma$.

Since $Y$ is a rational homology sphere, there exists a positive integer $k$, so that $k \omega(\mathbf{x}, \mathbf{y})$ is homologous to the sum of some copies of $\alpha$ - and $\beta$-curves. So there exists a 2-chain $\mathcal{D}$, such that $\partial \mathcal{D}$ consists of $k \omega(\mathbf{x}, \mathbf{y})$ and a linear combination of $\alpha-$ and $\beta$-curves. The following elementary lemma is important.

Lemma 4.2 With the notation as above, given $\mathbf{x}, \mathbf{y} \in \mathbb{T}_{\alpha} \cap \mathbb{T}_{\beta}$, we have

$$
\mathfrak{h}_{\mathbf{w}, \mathbf{z}}(\mathbf{x})-\mathfrak{h}_{\mathbf{w}, \mathbf{z}}(\mathbf{y})=\frac{1}{k} \sum_{i=1}^{l}\left(n_{z_{i}}(\mathcal{D})-n_{w_{i}}(\mathcal{D})\right) \operatorname{PD}\left[\mu_{i}\right]
$$

Proof We cap off the copies of $\alpha$ - and $\beta$-curves in $\partial \mathcal{D}$ to get a 2-dimensional chain $G \subset Y$, so that $\partial G=k \omega(\mathbf{x}, \mathbf{y}) . G \cap(Y-\operatorname{int}(\operatorname{Nd}(L)))$ is a homology between $k \omega(\mathbf{x}, \mathbf{y})$ and some copies of $\mu_{i}$ 's. And the coefficients of $\mu_{i}$ 's can be computed by counting the algebraic intersection numbers of $K_{i}$ with $\mathcal{D}$. Since $\underline{\mathfrak{s}}_{\mathbf{w}, \mathbf{z}}(\mathbf{x})-\underline{\mathfrak{s}}_{\mathbf{w}, \mathbf{z}}(\mathbf{y})=\operatorname{PD}([\omega(\mathbf{x}, \mathbf{y})])$ [13, Lemma 3.11], we have that

$$
\begin{aligned}
k\left(\mathfrak{h}_{\mathbf{w}, \mathbf{z}}(\mathbf{x})-\mathfrak{h}_{\mathbf{w}, \mathbf{z}}(\mathbf{y})\right) & =\frac{k}{2}\left(c_{1}\left(\underline{\mathfrak{s}}_{\mathbf{w}, \mathbf{z}}(\mathbf{x})\right)-c_{1}\left(\underline{\mathfrak{s}}_{\mathbf{w}, \mathbf{z}}(\mathbf{y})\right)\right) \\
& =k \operatorname{PD}([\omega(\mathbf{x}, \mathbf{y})]) \\
& =\operatorname{PD}\left(\sum_{i=1}^{l}\left(n_{z_{i}}(\mathcal{D})-n_{w_{i}}(\mathcal{D})\right)\left[\mu_{i}\right]\right) .
\end{aligned}
$$

Hence the result holds. 
The above lemma indicates that $\mathfrak{H}$ defines a $\mathbb{Q}^{l}$-grading on $\widehat{C F L}(Y, L)$. Following Rasmussen [15], we call this grading an Alexander grading. Given $h^{*} \in H^{2}(Y, L ; \mathbb{Q})$, let

$$
\widehat{C F L}\left(Y, L, h^{*}\right) \cong \bigoplus_{\mathfrak{r} \in \underline{\operatorname{Spin}^{c}}(Y, L), \mathfrak{H}(\mathfrak{r})=h^{*}} \widehat{C F L}(Y, L, \mathfrak{r}) .
$$

Then Lemma 4.1 implies that

$$
\widehat{C F L}\left(Y, L, h^{*}\right) \cong \widehat{C F L}\left(Y, L,-h^{*}\right)
$$

\subsection{A formula for split links}

The following formula for split links will be used in the proof of Theorem 1.1.

Proposition 4.3 Suppose $L_{1} \subset Y_{1}, L_{2} \subset Y_{2}$ are two oriented links in rational homology spheres, then $L=L_{1} \sqcup L_{2}$ is a split link in $Y=Y_{1} \# Y_{2}$. Let $\mathfrak{r}_{1} \in \operatorname{Spin}^{c}\left(Y_{1}, L_{1}\right)$, $\mathfrak{r}_{2} \in \operatorname{Spin}^{c}\left(Y_{2}, L_{2}\right)$, then they naturally give a relative $\operatorname{Spin}^{c}$ structure $\mathfrak{r}=\mathfrak{r}_{1} \# \mathfrak{r}_{2} \in$ $\operatorname{Spin}^{c}(Y, L)$. We have the following formula:

$$
\widehat{C F L}(Y, L, \mathfrak{r}) \cong \widehat{C F L}\left(Y_{1}, L_{1}, \mathfrak{r}_{1}\right) \otimes \widehat{C F L}\left(Y_{2}, L_{2}, \mathfrak{r}_{2}\right) \otimes \widehat{H F}\left(S^{1} \times S^{2}\right) .
$$

Proof Suppose $L_{i}$ has $l_{i}$ components, $i=1,2$. Let $\left(\Sigma_{i}, \boldsymbol{\alpha}_{i}, \boldsymbol{\beta}_{i}, \mathbf{w}_{i}, \mathbf{z}_{i}\right)$ be a weakly admissible balanced $2 l_{i}$-pointed Heegaard diagram associated to the pair $\left(Y_{i}, L_{i}\right)$. We construct a Heegaard diagram for $(Y, L)$ as follows.

Let $A=S^{1} \times[-1,1]$ be a tube, $\alpha_{0}=S^{1} \times 0$ is a belt circle, and $\beta_{0}$ is a small Hamiltonian perturbation of $\alpha_{0}$. Let $\Sigma=\Sigma_{1} \# \Sigma_{2}$, with $A$ as the neck of the connected sum. We put the feet of this tube into two regions which contain base points. We can verify that

$$
\left(\Sigma, \boldsymbol{\alpha}_{1} \cup\left\{\alpha_{0}\right\} \cup \boldsymbol{\alpha}_{2}, \boldsymbol{\beta}_{1} \cup\left\{\beta_{0}\right\} \cup \boldsymbol{\beta}_{2}, \mathbf{w}_{1} \cup \mathbf{w}_{2}, \mathbf{z}_{1} \cup \mathbf{z}_{2}\right)
$$

is a weakly admissible Heegaard diagram for $(Y, L)$.

Now the desired formula can be proved by a standard argument.

\section{Proof of the main theorem}

In this section, we are going to prove our main theorem. The idea of the proof is the same as in [14], but we will take a slightly different approach.

First of all, let us check Theorem 1.1 for certain knots in lens spaces. As in [9], if one does $(p / q)$-surgery on one component of the Hopf link, then the other component gives a knot $O_{p / q}$ in the lens space $L(p, q)$. The complement of $O_{p / q}$ is a solid torus, with a meridian disk $D_{p / q}$. Our result is: 
Lemma 5.1 There are exactly $p$ relative $\operatorname{Spin}^{c}$ structures satisfying

$$
\widehat{H F K}\left(L(p, q), O_{p / q}, \mathfrak{r}\right) \neq 0 .
$$

One can denote these relative Spinc structures by $\mathfrak{r}_{1}, \ldots, \mathfrak{r}_{p}$, so that

$$
\left\langle c_{1}\left(\mathfrak{r}_{i}\right),\left[D_{p / q}\right]\right\rangle=2 i-1 .
$$

Hence Theorem 1.1 holds for $O_{p / q}$.

Proof $\left(L(p, q), O_{p / q}\right)$ admits a genus-1 Heegaard diagram, such that $\mathbb{T}_{\alpha} \cap \mathbb{T}_{\beta}$ has exactly $p$ intersection points, which correspond to $p$ different relative $\operatorname{Spin}^{c}$ structures. As in $\left[9\right.$, Lemma 7.1], we can denote these relative $\operatorname{Spin}^{c}$ structures by $\mathfrak{r}_{1}, \ldots, \mathfrak{r}_{p}$, such that $\mathfrak{r}_{i+1}-\mathfrak{r}_{i}$ is the positive generator of $H^{2}\left(L(p, q), O_{p / q}\right) \cong \mathbb{Z}$, for $i=1, \ldots, p-1$. Let $a_{i}=\left\langle c_{1}\left(\mathfrak{r}_{i}\right),\left[D_{p / q}\right]\right\rangle$, then $a_{i+1}-a_{i}=2$.

Since

$$
\left\langle c_{1}(\mathfrak{r})+c_{1}(J(\mathfrak{r})+\operatorname{PD}[\mu]),\left[D_{p / q}\right]\right\rangle=\left\langle 2 \operatorname{PD}[\mu],\left[D_{p / q}\right]\right\rangle=2 p,
$$

by Lemma 4.1 , the set $\left\{a_{1}, a_{2}, \ldots, a_{p}\right\}$ admits an involution $a \mapsto 2 p-a$. Hence we must have $a_{i}=2 i-1$.

Now we can check Theorem 1.1 directly for $O_{p / q}$.

Suppose $L$ is an oriented link in a rational homology 3 -sphere $Y,(\Sigma, \boldsymbol{\alpha}, \boldsymbol{\beta}, \mathbf{w}, \mathbf{z})$ is a (generic) balanced $2 l-$ pointed Heegaard diagram associated to the pair $(Y, L)$. Given an integral class $h \in H_{2}(Y, L ; \mathbb{Q})$, let

$$
\mathcal{F}_{\mathbf{w}, \mathbf{z}}^{h}(\mathbf{x})=\left\langle\mathfrak{h}_{\mathbf{w}, \mathbf{z}}(\mathbf{x}), h\right\rangle,
$$

for any $\mathbf{x} \in \mathbb{T}_{\alpha} \cap \mathbb{T}_{\beta}$. Thus $\mathcal{F}_{\mathbf{w}, \mathbf{z}}^{h}$ defines a $\mathbb{Q}$-grading on $\widehat{C F L}(Y, L)$.

Proposition 5.2 Suppose $L$ is a null-homologous oriented link, and $F$ is a minimal genus Seifert surface of $L$. Then Theorem 1.1 holds for $h=[F]$.

Proof As in [13], we can get a Heegaard diagram

$$
\left(\Sigma^{\prime}, \boldsymbol{\alpha}^{\prime}, \boldsymbol{\beta}^{\prime}, w_{1}, z_{l}\right)
$$

for $(\kappa(Y), \kappa(L))$, by adding tubes with feet at $z_{i}$ and $w_{i+1}$, for $i=1, \ldots, l-1$. Suppose $\mathcal{D}$ is a topological disk in $\operatorname{Sym}^{g+l-1}\left(\Sigma^{\prime}\right), \partial \mathcal{D} \subset \mathbb{T}_{\alpha} \cup \mathbb{T}_{\beta}$, then $n_{z_{i}}(\mathcal{D})=$ $n_{w_{i+1}}(\mathcal{D})$. Hence

$$
n_{z_{1}}(\mathcal{D})-n_{w_{l}}(\mathcal{D})=\sum_{i=1}^{l}\left(n_{z_{i}}(\mathcal{D})-n_{w_{i}}(\mathcal{D})\right) .
$$


By Lemma 4.2, we conclude that the $\mathbb{Q}$-grading defined by $\mathcal{F}_{\mathbf{w}, \mathbf{Z}}^{[F]}$ coincides with the usual Alexander $\mathbb{Z}$-grading defined on $\widehat{C F K}(\kappa(Y), \kappa(L))$, as relative gradings.

The proof of [13, Theorem 1.1] shows that

$$
\widehat{H F L}(Y, L) \cong \widehat{H F K}(Y, L)
$$

as relative $\mathbb{Q}$-graded $\mathbb{F}_{2}$-vector spaces. Moreover, by Lemma 4.1, $\widehat{H F L}(Y, L)$, equipped with the absolute $\mathbb{Q}$-grading given by $\mathcal{F}_{\mathbf{w}, \mathbf{z}}^{[F]}$, is symmetric with respect to the origin 0 . Hence this absolute $\mathbb{Q}$-grading is identical to the usual absolute Alexander $\mathbb{Z}$-grading on $\widehat{H F K}(Y, L)$.

Now we can apply Theorem 3.1 to get the conclusion.

In order to reduce the general case to the case of $h=[F]$, we are going to use the "cabling trick" introduced in [14]. The idea is to consider a $(\mathbf{p}, \mathbf{q})$-cable of $L$, hence to reduce the general case to the case treated in Proposition 5.2. The method of dealing with cables comes from Hedden's work [4].

Suppose $L$ is an $l$-component oriented link in $Y$, the components of $L$ are denoted by $K_{1}, \ldots, K_{l}$. Using stabilizations and handle-slides if necessary, we can construct a $2 l$-pointed Heegaard diagram $(\Sigma, \boldsymbol{\alpha}, \boldsymbol{\beta}, \mathbf{w}, \mathbf{z})$ associated to the pair $(Y, L)$, satisfying the following conditions:

(1) For each $i \in\{1,2, \ldots, l\}, \beta_{i}$ represents a meridian for $K_{i}$, namely, $w_{i}$ and $z_{i}$ lie on a curve $\lambda_{i}$ which meets $\beta_{i}$ in a single point, and is disjoint from all the other $\beta$-curves.

(2) The curve $\beta_{i}$ meets $\alpha_{i}$ transversely in a single point, and is disjoint from all the other $\alpha$-curves.

The curve $\lambda_{i} \subset \Sigma$ is isotopic to $K_{i}$ in $Y$, hence $\Sigma$ specifies a frame of $K_{i}$.

Suppose $\mathbf{p}=\left(p_{1}, \ldots, p_{l}\right), \mathbf{q}=\left(q_{1}, \ldots, q_{l}\right)$ are two $l$-tuples of positive integers, where

$$
q_{i}=p_{i} n_{i}+1
$$

for some $l$-tuple of positive integers $\mathbf{n}=\left(n_{1}, \ldots, n_{l}\right)$. We replace $\beta_{i}$ with a new curve $\gamma_{i}$, gotten by performing a "finger move" of $\beta_{i}$ along $\lambda_{i}$ with multiplicity $\left(p_{i}-1\right)$, and then winding $n_{i}$ times parallel to $\beta_{i}$. We put a new basepoint $z_{i}^{\prime}$ inside the end of the finger. The new diagram $\left(\Sigma, \boldsymbol{\alpha}, \boldsymbol{\gamma}, \mathbf{w}, \mathbf{z}^{\prime}\right)$ gives the link $C(L)=C_{\mathbf{p}, \mathbf{q}}(L)$, which is the $(\mathbf{p}, \mathbf{q})$-cable of $L$ with respect to the frame specified by $\Sigma$. We can also find a basepoint $t_{i}$ outside the finger, so that $(\Sigma, \boldsymbol{\alpha}, \boldsymbol{\gamma}, \mathbf{w}, \mathbf{t})$ describes $L$. See Figure 1 for an illustration of the local diagram. 


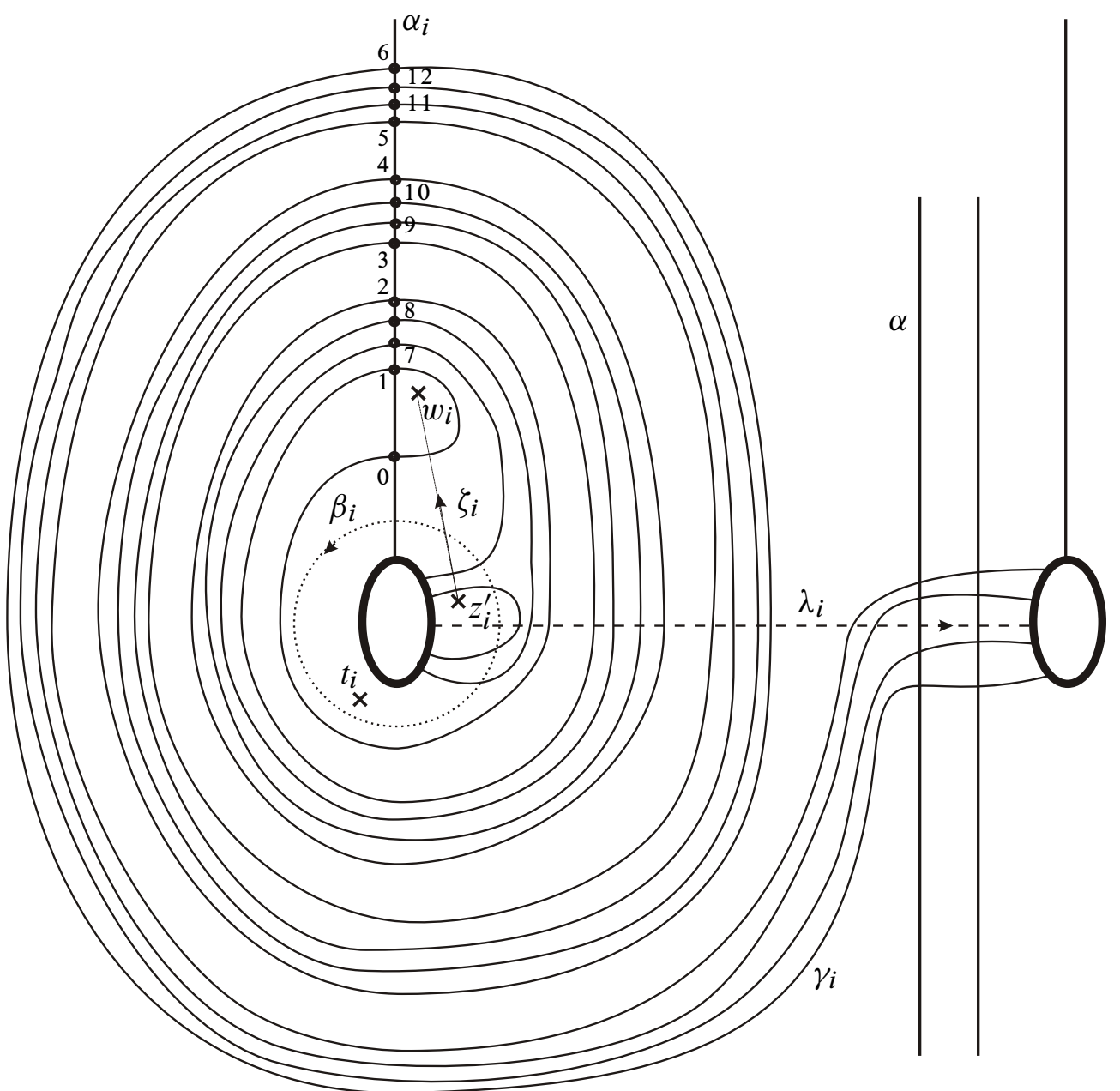

Figure 1: The local Heegaard diagram for a $(3,10)-$ cable

We oriented the curves $\beta_{i}, \lambda_{i}$ as indicated in Figure 1. The curve $\gamma_{i}$ is also equipped with an orientation induced from the orientation on $\beta_{i}$.

Let $\operatorname{Nd}\left(\lambda_{i}\right)$ be a regular neighborhood of $\lambda_{i}$ inside which we perform the finger move. Let $\operatorname{Nd}\left(\beta_{i}\right)$ be a regular neighborhood of $\beta_{i}$ inside which we perform the winding. An intersection point of $\gamma_{i}$ with an $\alpha$-curve is exterior, if it lies in $\operatorname{Nd}\left(\beta_{i}\right)$. (In this case, the only possibility for this $\alpha$-curve is $\alpha_{i}$.) An intersection point of $\gamma_{i}$ with an $\alpha$-curve is interior, if it lies in $\operatorname{Nd}\left(\lambda_{i}\right)$. An intersection point $\mathbf{x} \in \mathbb{T}_{\alpha} \cap \mathbb{T}_{\gamma}$ is called an exterior intersection point, if its $\gamma_{i}$-component is exterior for all $i=1, \ldots, l$. 
Let $\iota: Y-L \rightarrow Y-C(L)$ be the inclusion map, $\iota_{*}: H_{1}(Y-L) \rightarrow H_{1}(Y-C(L))$, $\iota^{*}: H^{2}(Y, C(L)) \rightarrow H^{2}(Y, L)$ be the induced maps on (co)homologies. Let $\mu_{i}^{\prime}$ be the meridian of $C_{p_{i}, q_{i}}\left(K_{i}\right)$. Then $\iota_{*}\left(\left[\mu_{i}\right]\right)=p_{i}\left[\mu_{i}^{\prime}\right]$.

Inside $\operatorname{Nd}\left(\beta_{i}\right)$, the curve $\alpha_{i}$ has $2\left(p_{i}-1\right) n_{i}+1$ (exterior) intersection points with $\gamma_{i}$. We define a function

$$
S_{j}: \alpha_{i} \cap \gamma_{i} \cap \operatorname{Nd}\left(\beta_{i}\right) \rightarrow \mathbb{Q},
$$

which is uniquely characterized up to an overall translation as follows.

Given $x, y \in \alpha_{i} \cap \gamma_{i} \cap \operatorname{Nd}\left(\beta_{i}\right)$, there are two arcs $a \subset \alpha_{i}$ and $b \subset \gamma_{i}$, both connecting $x$ to $y$, so that $a-b$ is rationally homologous to a rational linear combination of $\alpha$-curves and $\gamma$-curves. Let $\mathcal{D}$ be a 2 -chain, such that $\partial \mathcal{D}$ consists of $a-b$ and a rational linear combination of $\alpha$-curves and $\gamma$-curves, then $S_{j}$ satisfies

$$
S_{j}(x)-S_{j}(y)=n_{z_{j}^{\prime}}(\mathcal{D})-n_{w_{j}}(\mathcal{D}) .
$$

Draw an oriented arc $\zeta_{j} \subset \Sigma$ connecting $z_{j}^{\prime}$ to $w_{j}$ (see Figure 1), then we also have

$$
S_{j}(x)-S_{j}(y)=\zeta_{j} \cdot \partial \mathcal{D} .
$$

Definition 5.3 Suppose $K, L$ are two disjoint knots in a rational homology sphere $Z$. Let $\partial_{*}: H_{2}(Z, K ; \mathbb{Q}) \rightarrow H_{1}(K ; \mathbb{Q})$ be the boundary map. Since $Z$ is a rational homology sphere, $\partial_{*}$ is an isomorphism. The linking number of $K, L$ is defined to be

$$
\partial_{*}^{-1}([K]) \cdot[L] \in \mathbb{Q},
$$

denoted by $\operatorname{lk}(K, L)$. Suppose $\lambda$ is a frame on $K$, then $\lambda$ can be viewed as a knot on $\partial \operatorname{Nd}(K)$. We call $\operatorname{lk}(K, \lambda)$ the self-linking number of $K$ with frame $\lambda$, denoted by $\operatorname{lk}(\lambda, \lambda)$.

Lemma 5.4 (Compare [14, Lemma 3.7].) (1) We can label the $2\left(p_{i}-1\right) n_{i}+1$ points in $\alpha_{i} \cap \gamma_{i} \cap \mathrm{Nd}\left(\beta_{i}\right)$ by

$$
x_{i}^{0}, x_{i}^{1}, \ldots, x_{i}^{2\left(p_{i}-1\right) n_{i}},
$$

such that

$$
S_{i}\left(x_{i}^{k}\right)-S_{i}\left(x_{i}^{k+1}\right)= \begin{cases}1, & \text { if } k=0, \\ \frac{k}{2 n_{i}}+1+p_{i} \cdot \operatorname{lk}\left(\lambda_{i}, \lambda_{i}\right), & \text { if } 2 n_{i} \mid k \text { and } k>0, \\ \left\lfloor\frac{k}{2 n_{i}}\right\rfloor+1, & \text { if } 2 n_{i} \nmid k \text { and } k \text { is even, } \\ p_{i}-\left\lfloor\frac{k}{2 n_{i}}\right\rfloor-1, & \text { if } 2 n_{i} \nmid k \text { and } k \text { is odd. }\end{cases}
$$


In particular,

$$
S_{i}\left(x_{i}^{0}\right)-S_{i}\left(x_{i}^{2 n_{i}}\right)=p_{i} n_{i} .
$$

(2) For $i \neq j$ we have that

$$
S_{j}\left(x_{i}^{k}\right)-S_{j}\left(x_{i}^{k+1}\right)= \begin{cases}p_{j} \cdot \operatorname{lk}\left(K_{i}, K_{j}\right), & \text { if } 2 n_{i} \mid k, \\ 0, & \text { otherwise. }\end{cases}
$$

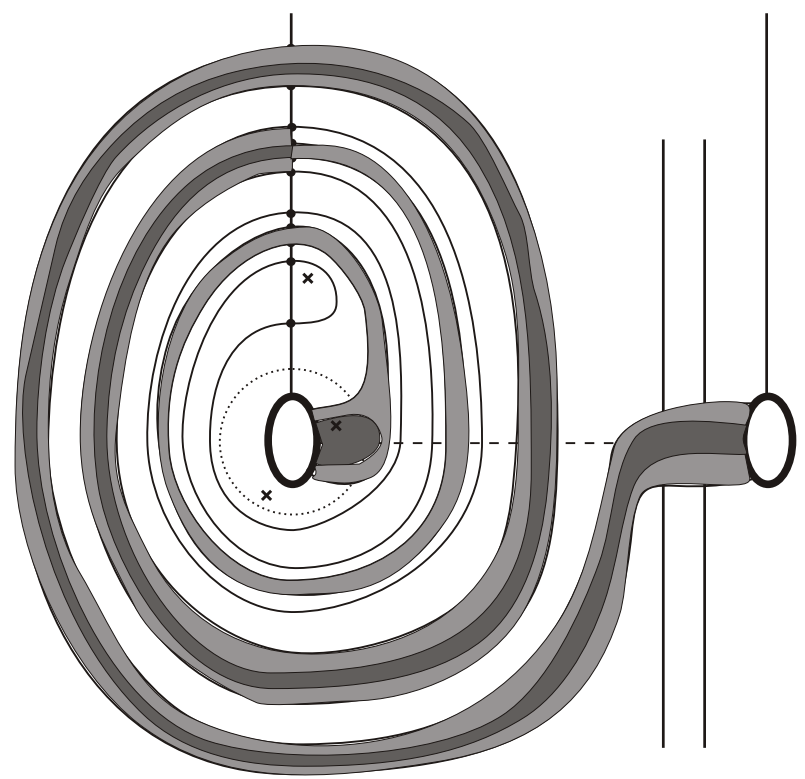

Figure 2: A disk connecting $x^{3}$ to $x^{4}$. The dark grey region has multiplicity 2 while the light grey region has multiplicity 1 .

Proof (1) We suppress the subscript $i$ when there is no ambiguity. The labelling of the $2(p-1) n+1$ points is illustrated in Figure 1 .

The two points $x^{0}$ and $x^{1}$ are connected by a bigon with $n_{z^{\prime}}=0, n_{w}=-1$, which implies that $S\left(x^{0}\right)-S\left(x^{1}\right)=1$.

When $k>0$, suppose $k=h \cdot(2 n)+r$, where $0 \leq h \leq p-2,0 \leq r \leq 2 n-1$. If $r$ is odd, then there is a bigon connecting $x^{k}$ to $x^{k+1}$, with $n_{z^{\prime}}=p-h-1, n_{w}=0$. See Figure 2 for an illustration. Using (3), we conclude that $S\left(x^{k}\right)-S\left(x^{k+1}\right)=p-h-1$ when $k$ is odd.

When $r \in\{1, \ldots, 2 n-2\}$, there is an arc $a_{k, k+2} \subset \alpha_{i}$ and an $\operatorname{arc} b_{k, k+2} \subset \gamma_{i}$, both connecting $x^{k}$ to $x^{k+2}$, such that $a_{k, k+2}-b_{k, k+2}$ is homologous to $-\beta_{i}$ in $\Sigma$. See 
Figure 3 for an illustration. It follows that $a_{k, k+2}-b_{k, k+2}+\gamma_{i}$ bounds a domain in $\Sigma$. Since $a_{k, k+2}$ and $b_{k, k+2}$ does not intersect the arc $\zeta_{i}$ shown in Figure 1, using (4), $S\left(x^{k}\right)-S\left(x^{k+2}\right)$ is calculated by $\zeta_{i} \cdot \gamma_{i}=p$. Thus $S\left(x^{k}\right)-S\left(x^{k+2}\right)=p$. Combined with the last paragraph, we see that $S\left(x^{k}\right)-S\left(x^{k+1}\right)=h+1$ when $k$ is even and $2 n \nmid k$.

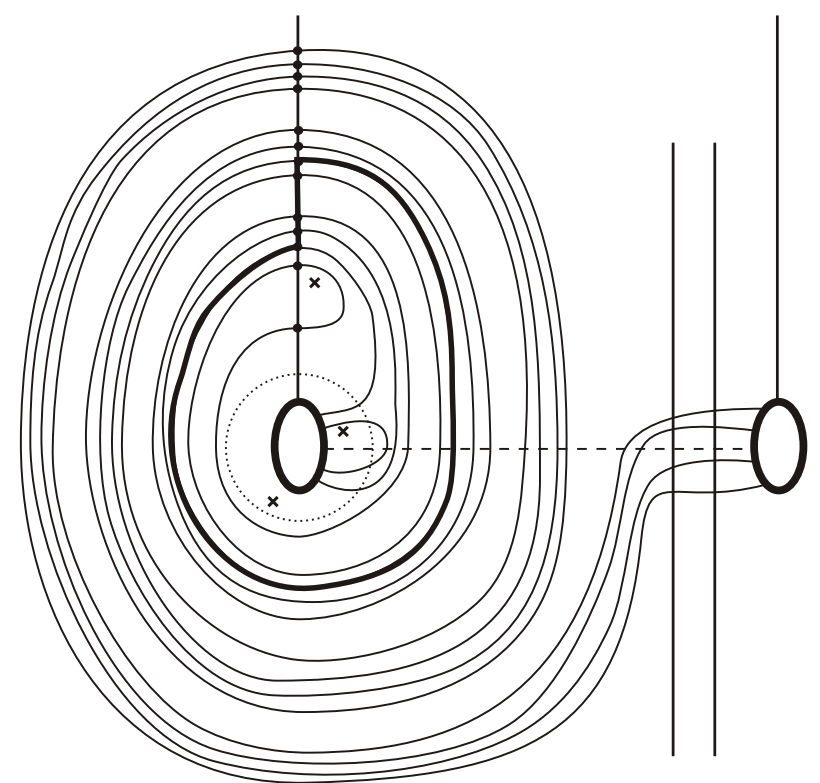

Figure 3: Two arcs connecting $x^{7}$ to $x^{9}$

When $2 n \mid k$, as in Figure 4, there is an arc $a_{k-1, k+1} \subset \alpha_{i}$ and an arc $b_{k-1, k+1} \subset \gamma_{i}$, both connecting $x^{k-1}$ to $x^{k+1}$, such that $a_{k-1, k+1}-b_{k-1, k+1}$ is homologous to $-\beta_{i}-\lambda_{i}$. So $a_{k-1, k+1}-b_{k-1, k+1}+\gamma_{i}+\lambda_{i}$ bounds a $2-$ chain $\mathcal{D}_{1}$. Since $Y$ is a rational homology sphere, $\lambda_{i}$ cobounds a 2 -chain $\mathcal{D}_{2}$ with some copies of $\alpha$-curves and $\gamma$-curves, where the multiplicity of $\gamma_{i}$ in $\partial \mathcal{D}_{2}$ is $-\operatorname{lk}\left(\lambda_{i}, \lambda_{i}\right)$. So $\mathcal{D}_{1}-\mathcal{D}_{2}$ is a 2-chain bounded by $a_{k-1, k+1}-b_{k-1, k+1}$ and some copies of $\alpha$-curves and $\gamma-$ curves, where the multiplicity of $\gamma_{i}$ is $1+\operatorname{lk}\left(\lambda_{i}, \lambda_{i}\right)$. Using (4), $S\left(x^{k-1}\right)-S\left(x^{k+1}\right)$ is calculated by

$$
\zeta_{i} \cdot\left(-b_{k-1, k+1}+\left(1+\operatorname{lk}\left(\lambda_{i}, \lambda_{i}\right)\right) \gamma_{i}\right)=1+p+p \cdot \operatorname{lk}\left(\lambda_{i}, \lambda_{i}\right) .
$$

Since $S\left(x^{k-1}\right)-S\left(x^{k}\right)=p-\lfloor(k-1) /(2 n)\rfloor-1=p-k /(2 n)$, we have

$$
S\left(x^{k}\right)-S\left(x^{k+1}\right)=\frac{k}{2 n}+1+p \cdot \operatorname{lk}\left(\lambda_{i}, \lambda_{i}\right)
$$


(2) The proof is similar to (1). We note that, when $2 n_{i} \nmid k$ the domain involved is always supported in a neighborhood of $\alpha_{i} \cup \beta_{i} \cup \lambda_{i}$. So both $n_{z_{j}^{\prime}}$ and $n_{w_{j}}$ are zero, hence $S_{j}\left(x_{i}^{k}\right)-S_{j}\left(x_{i}^{k+1}\right)=0$.

When $2 n_{i} \mid k$, as in (1), the multiplicity of $\gamma_{j}$ in the boundary of $\mathcal{D}_{1}-\mathcal{D}_{2}$ is $\operatorname{lk}\left(K_{i}, K_{j}\right)$. So we have

$$
S_{j}\left(x_{i}^{k}\right)-S_{j}\left(x_{i}^{k+1}\right)=S_{j}\left(x_{i}^{k-1}\right)-S_{j}\left(x_{i}^{k+1}\right)=p_{j} \cdot \operatorname{lk}\left(K_{i}, K_{j}\right) .
$$

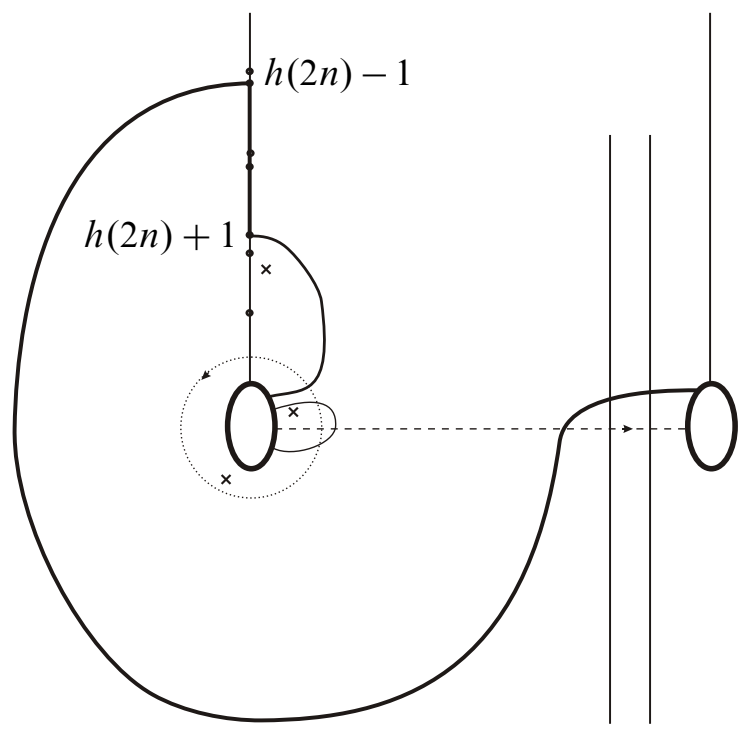

Figure 4: Two arcs connecting $x^{h(2 n)-1}$ to $x^{h(2 n)+1}$

Corollary 5.5 If $\operatorname{lk}\left(\lambda_{i}, \lambda_{i}\right) \geq 0$, then we always have

$$
S_{i}\left(x_{i}^{k}\right)>S_{i}\left(x_{i}^{k+1}\right) \text {. }
$$

Proof This is a simple consequence of Lemma 5.4 (1).

Convention 5.6 From now on, we always choose the frames $\lambda_{i}, i=1, \ldots, l$, such that $\operatorname{lk}\left(\lambda_{i}, \lambda_{i}\right) \geq 0$ for each $i$.

Definition 5.7 We observe that the point $x_{i}^{0}$ comes from the original intersection point $\alpha_{i} \cap \beta_{i}$. The points $x_{i}^{0}, x_{i}^{1}, \ldots, x_{i}^{2 n_{i}}$ are called outermost points. If $\mathbf{x}$ is an exterior intersection point, and the $\gamma_{i}$-component of $\mathbf{x}$ is outermost for all $i$, then $\mathbf{x}$ is called an outermost exterior point. 
Definition 5.8 Let $A$ be a subset of $\{1, \ldots, l\}$. If the $\gamma_{i}$-coordinate of an intersection point $\mathbf{u} \in \mathbb{T}_{\alpha} \cap \mathbb{T}_{\gamma}$ is supported in $\operatorname{Nd}\left(\lambda_{i}\right)$ when $i \in A$, and is $x_{i}^{0}$ when $i \notin A$, then $\mathbf{u}$ is called a type- $A$ interior intersection point. The set of type $-A$ interior intersection points is denoted by $\mathrm{I}^{A}$. Given an intersection point $\mathbf{x} \in \mathbb{T}_{\alpha} \cap \mathbb{T}_{\beta}$, one can associate to it a corresponding intersection point $\mathbf{x}^{A} \in \mathbb{T}_{\alpha} \cap \mathbb{T}_{\gamma}$, so that the $\gamma_{i}$-coordinate of $\mathbf{x}^{A}$ is $x_{i}^{2 n_{i}}$ when $i \in A$, is $x_{i}^{0}$ when $i \notin A$, and all other coordinates are the same as the coordinates of $\mathbf{x}$. When $A=\varnothing, \mathbf{x}^{\varnothing}$ is also denoted by $\mathbf{x}^{\prime}$.

In order to emphasize the dependence of the diagram on $\mathbf{n}$, we sometimes put a subscript (n) in the notation. For example, the base points $\mathbf{z}^{\prime}$ are denoted by $\mathbf{z}_{(\mathbf{n})}^{\prime}$, and the set of type- $A$ interior intersection points is denoted by $\mathrm{I}_{(\mathbf{n})}^{A}$.

For two different $\mathbf{n}_{1}, \mathbf{n}_{2}$, there is a natural 1-1 correspondence between $\mathrm{I}_{\left(\mathbf{n}_{1}\right)}^{A}$ and $\mathrm{I}_{\left(\mathbf{n}_{2}\right)}^{A}$. Suppose $\mathbf{u}_{\left(\mathbf{n}_{1}\right)} \in \mathrm{I}_{\left(\mathbf{n}_{1}\right)}^{A}$, then the corresponding point in $\mathrm{I}_{\left(\mathbf{n}_{2}\right)}^{A}$ is denoted by $\mathbf{u}_{\left(\mathbf{n}_{2}\right)}$. Let

$$
\begin{aligned}
\mathfrak{h}_{\mathbf{w}, \mathbf{z}}: & \mathbb{T}_{\alpha} \cap \mathbb{T}_{\beta} \rightarrow H^{2}(Y, L ; \mathbb{Q}), \\
\mathfrak{h}_{\mathbf{w}, \mathbf{t}}: & \mathbb{T}_{\alpha} \cap \mathbb{T}_{\gamma} \rightarrow H^{2}(Y, L ; \mathbb{Q}), \\
\mathfrak{h}_{\mathbf{w}, \mathbf{z}^{\prime}}: & \mathbb{T}_{\alpha} \cap \mathbb{T}_{\gamma} \rightarrow H^{2}(Y, C(L) ; \mathbb{Q}),
\end{aligned}
$$

be the affine maps defined in Section 4.4.

The following observation is important:

Lemma 5.9 Fix a point $\mathbf{x} \in \mathbb{T}_{\alpha} \cap \mathbb{T}_{\beta}$, a set $A \subset\{1, \ldots, l\}$ and a point $\mathbf{u}_{(\mathbf{n})} \in \mathrm{I}_{(\mathbf{n})}^{A}$. Then

$$
\mathfrak{h}_{\mathbf{w}, \mathbf{z}_{(\mathbf{n})}^{\prime}}\left(\mathbf{x}_{(\mathbf{n})}^{A}\right)-\mathfrak{h}_{\mathbf{w}, \mathbf{z}_{(\mathbf{n})}^{\prime}}\left(\mathbf{u}_{(\mathbf{n})}\right)
$$

is a constant independent of $\mathbf{n}$.

Proof Given two $l$-tuples $\mathbf{n}_{1}, \mathbf{n}_{2}$, without loss of generality, we can assume $\mathbf{n}_{1}<\mathbf{n}_{2}$, that is, every coordinate of $\mathbf{n}_{1}$ is less than or equal to the corresponding coordinate of $\mathbf{n}_{2}$, and at least one equality does not hold. Suppose $\mathcal{D}_{\left(\mathbf{n}_{1}\right)}$ is a 2-chain whose boundary consists of $k \omega\left(\mathbf{x}_{\left(\mathbf{n}_{1}\right)}^{A}, \mathbf{u}_{\left(\mathbf{n}_{1}\right)}\right)$ and a sum of some copies of $\alpha$-curves and $\gamma_{\left(\mathbf{n}_{1}\right)}$-curves. Then we can get a domain $\mathcal{D}_{\left(\mathbf{n}_{2}\right)}$ by performing finger moves to $\mathcal{D}_{\left(\mathbf{n}_{1}\right)}$, so that $\mathcal{D}_{\left(\mathbf{n}_{2}\right)}$ is the corresponding domain for $\mathbf{x}_{\left(\mathbf{n}_{2}\right)}^{A}, \mathbf{u}_{\left(\mathbf{n}_{2}\right)}$.

When $i \in A$, we have $n_{z_{i(\mathbf{n})}^{\prime}}\left(\mathcal{D}_{(\mathbf{n})}\right)-n_{w_{i}}\left(\mathcal{D}_{(\mathbf{n})}\right)=\zeta_{i} \cdot \partial \mathcal{D}_{(\mathbf{n})}$. Since the $\gamma_{i}$-coordinate of $\mathbf{u}_{(\mathbf{n})}$ is supported in $\operatorname{Nd}\left(\lambda_{i}\right)$, and the $\gamma_{i}$-coordinate of $\mathbf{x}_{(\mathbf{n})}^{A}$ is $x_{(\mathbf{n})}^{2 n_{i}}$, the finger moves do not change $\zeta_{i} \cdot \partial \mathcal{D}_{(\mathbf{n})}$.

When $i \notin A$, the $\gamma_{i}$-coordinates of $\mathbf{x}_{(\mathbf{n})}^{A}$ and $\mathbf{u}_{(\mathbf{n})}$ are both $x_{i(\mathbf{n})}^{0}$. The finger moves do not change $n_{z_{i(\mathbf{n})}^{\prime}}\left(\mathcal{D}_{(\mathbf{n})}\right)-n_{w_{i}}\left(\mathcal{D}_{(\mathbf{n})}\right)$. Thus our desired result holds by Lemma 4.2. $\square$ 
Lemma 5.10 Given $\mathbf{x}, \mathbf{y} \in \mathbb{T}_{\alpha} \cap \mathbb{T}_{\beta}$, we have

$$
\mathfrak{h}_{\mathbf{w}, \mathbf{z}}(\mathbf{x})-\mathfrak{h}_{\mathbf{w}, \mathbf{z}}(\mathbf{y})=\mathfrak{h}_{\mathbf{w}, \mathbf{t}}\left(\mathbf{x}^{\prime}\right)-\mathfrak{h}_{\mathbf{w}, \mathbf{t}}\left(\mathbf{y}^{\prime}\right)=\iota^{*}\left(\mathfrak{h}_{\mathbf{w}, \mathbf{z}^{\prime}}\left(\mathbf{x}^{\prime}\right)-\mathfrak{h}_{\mathbf{w}, \mathbf{z}^{\prime}}\left(\mathbf{y}^{\prime}\right)\right)
$$

Proof It is obvious that

$$
\mathfrak{h}_{\mathbf{w}, \mathbf{z}}(\mathbf{x})-\mathfrak{h}_{\mathbf{w}, \mathbf{z}}(\mathbf{y})=\mathfrak{h}_{\mathbf{w}, \mathbf{t}}\left(\mathbf{x}^{\prime}\right)-\mathfrak{h}_{\mathbf{w}, \mathbf{t}}\left(\mathbf{y}^{\prime}\right)
$$

Suppose $\mathcal{D}$ is a domain, $\partial \mathcal{D}$ is the sum of $k \omega(\mathbf{x}, \mathbf{y})$ and some copies of $\alpha$ and $\beta$ curves. Then after applying finger moves and windings to $\mathcal{D}$, we get a domain $\mathcal{D}^{\prime}$, so that $\partial \mathcal{D}^{\prime}$ is the sum of $k \omega\left(\mathbf{x}^{\prime}, \mathbf{y}^{\prime}\right)$ and some copies of $\alpha$ and $\gamma$ curves. We can observe that

$$
n_{z_{i}^{\prime}}\left(\mathcal{D}^{\prime}\right)-n_{w_{i}}\left(\mathcal{D}^{\prime}\right)=p_{i}\left(n_{t_{i}}\left(\mathcal{D}^{\prime}\right)-n_{w_{i}}\left(\mathcal{D}^{\prime}\right)\right) .
$$

Hence

$$
\iota^{*}\left(\mathfrak{h}_{\mathbf{w}, \mathbf{z}^{\prime}}\left(\mathbf{x}^{\prime}\right)-\mathfrak{h}_{\mathbf{w}, \mathbf{z}^{\prime}}\left(\mathbf{y}^{\prime}\right)\right)=\mathfrak{h}_{\mathbf{w}, \mathbf{t}}\left(\mathbf{x}^{\prime}\right)-\mathfrak{h}_{\mathbf{w}, \mathbf{t}}\left(\mathbf{y}^{\prime}\right)
$$

by Lemma 4.2 and the fact that $\iota^{*}\left(p_{i} \operatorname{PD}\left(\left[\mu_{i}^{\prime}\right]\right)\right)=\operatorname{PD}\left(\left[\mu_{i}\right]\right)$.

Suppose $\mathbf{x}^{r}, \mathbf{x}^{s} \in \mathbb{T}_{\alpha} \cap \mathbb{T}_{\gamma}$ differ only at the $\gamma_{i}$-coordinate, where the coordinate of $\mathbf{x}^{r}$ is $x_{i}^{r}$, and the coordinate of $\mathbf{x}^{s}$ is $x_{i}^{s}$. From the definition of $S_{i}$ and Lemma 4.2, we conclude that

$$
\mathfrak{h}_{\mathbf{w}, \mathbf{z}^{\prime}}\left(\mathbf{x}^{r}\right)-\mathfrak{h}_{\mathbf{w}, \mathbf{z}^{\prime}}\left(\mathbf{x}^{s}\right)=\sum_{j=1}^{l}\left(S_{j}\left(x_{i}^{r}\right)-S_{j}\left(x_{i}^{s}\right)\right) \operatorname{PD}\left(\left[\mu_{j}^{\prime}\right]\right) .
$$

Now we fix an integral class $h \in H_{2}(Y, L ; \mathbb{Q})$, which satisfies $h \cdot\left[\mu_{i}\right]>0$ for each $i$. Suppose $F \subset M=Y-\operatorname{int}(\operatorname{Nd}(L))$ is a surface representing $h, F$ has no sphere components, and $\chi(F)$ is maximal among all such surfaces. We can assume $\partial F \cap \partial \mathrm{Nd}\left(K_{i}\right)$ consists of parallel oriented circles. Then $\partial F \cap \partial \operatorname{Nd}\left(K_{i}\right)$ is a torus link $T\left(P_{i}, Q_{i}\right)$, with respect to the frame specified by $\Sigma$.

Convention 5.11 From now on, we assume $p_{i} / P_{i}$ is an integer independent of $i$, say, $p_{i}=m P_{i}$. Then $C_{\mathbf{p}, \mathbf{q}}(L)$ is a null-homologous link.

Construction 5.12 A minimal genus Seifert surface $F^{\prime}$ for $C(L)$ can be obtained as follows. Inside the cable space $\operatorname{Nd}\left(K_{i}\right)-\operatorname{int}\left(\operatorname{Nd}\left(C_{p_{i}, q_{i}}\left(K_{i}\right)\right)\right)$, one can choose a properly embedded, Thurston norm minimizing surface $G_{i}$, so that $\partial G_{i} \cap \partial \mathrm{Nd}\left(K_{i}\right)$ is the torus link $T\left(m P_{i}, m Q_{i}\right)$, and $\partial G_{i} \cap \partial \mathrm{Nd}\left(C_{p_{i}, q_{i}}\left(K_{i}\right)\right)$ is a longitude of $C_{p_{i}, q_{i}}\left(K_{i}\right)$. Then $F^{\prime}$ is the union of $G_{1}, \ldots, G_{l}$ and $m$ parallel copies of $F$. A standard argument (see, for example, [14, Lemma 3.2]) in 3-dimensional topology shows that $F^{\prime}$ is a minimal genus Seifert surface for $C(L)$. Let $h^{\prime}=\left[F^{\prime}\right] \in H_{2}(Y, C(L))$. 
Recall the function

$$
\mathcal{F}_{\mathbf{w}, \mathbf{z}}^{h}: \mathbb{T}_{\alpha} \cap \mathbb{T}_{\beta} \rightarrow \mathbb{Q}
$$

is defined as

$$
\mathcal{F}_{\mathbf{w}, \mathbf{z}}^{h}(\mathbf{x})=\left\langle\mathfrak{h}_{\mathbf{w}, \mathbf{z}}(\mathbf{x}), h\right\rangle .
$$

Then $\mathcal{F}_{\mathbf{w}, \mathbf{z}}^{h}$ specifies an Alexander $\mathbb{Q}$-grading on $\widehat{C F L}(Y, L)$. We also equip the complex $\widehat{C F L}(Y, C(L))$ with the $\mathbb{Q}$-grading defined by $\mathcal{F}_{\mathbf{w}, \mathbf{z}^{\prime}}^{h^{\prime}}$

Convention 5.13 From now on, when we talk about the (1-dimensional) Alexander grading of $\widehat{C F L}(Y, L)$ and $\widehat{C F L}(Y, C(L))$, we always refer to the gradings defined by $\mathcal{F}_{\mathbf{w}, \mathbf{z}}^{h}$ and $\mathcal{F}_{\mathbf{w}, \mathbf{z}^{\prime}}^{h^{\prime}}$, respectively.

Let $D_{\text {top }}$ be the maximal grading such that the summand of $\widehat{H F L}(Y, L)$ at this grading is nontrivial. Similarly, let $D_{\text {top }}^{\prime}$ be the maximal grading such that the summand of $\widehat{H F L}(Y, C(L))$ at this grading is nontrivial. If the grading of $\mathbf{x}$ is no more than the grading of $\mathbf{y}$, then we denote as $\mathbf{x} \preceq \mathbf{y}$, and $\mathbf{x} \prec \mathbf{y}$ if their gradings are not equal.

Given $i \in\{1, \ldots, l\}$, suppose $\mathbf{x}^{r}, \mathbf{x}^{s} \in \mathbb{T}_{\alpha} \cap \mathbb{T}_{\gamma}$ are two points differing only at the $\gamma_{i}$-component, where their components are $x_{i}^{r}, x_{i}^{s}$, respectively. By (5), we have

$$
\mathcal{F}_{\mathbf{w}, \mathbf{z}^{\prime}}^{h^{\prime}}\left(\mathbf{x}^{r}\right)-\mathcal{F}_{\mathbf{w}, \mathbf{z}^{\prime}}^{h^{\prime}}\left(\mathbf{x}^{s}\right)=\sum_{j=1}^{l}\left(S_{j}\left(x_{i}^{r}\right)-S_{j}\left(x_{i}^{s}\right)\right) .
$$

In particular, if $r=0, s \geq 2 n_{i}$, then by Lemma 5.4, Corollary 5.5 and (6) we have

$$
\mathcal{F}_{\mathbf{w}, \mathbf{z}^{\prime}}^{h^{\prime}}\left(\mathbf{x}^{0}\right)-\mathcal{F}_{\mathbf{w}, \mathbf{z}^{\prime}}^{h^{\prime}}\left(\mathbf{x}^{s}\right) \geq p_{i} n_{i}-\left|p_{i}-2\right| \sum_{j \neq i} p_{j} \cdot\left|\operatorname{lk}\left(K_{i}, K_{j}\right)\right| .
$$

If $\mathbf{x}, \mathbf{y} \in \mathbb{T}_{\alpha} \cap \mathbb{T}_{\beta}$ are two intersection points, then by Lemma 5.10 and Construction 5.12, we have

$$
\mathcal{F}_{\mathbf{w}, \mathbf{z}^{\prime}}^{h^{\prime}}\left(\mathbf{x}^{\prime}\right)-\mathcal{F}_{\mathbf{w}, \mathbf{z}^{\prime}}^{h^{\prime}}\left(\mathbf{y}^{\prime}\right)=m\left(\mathcal{F}_{\mathbf{w}, \mathbf{z}}^{h}(\mathbf{x})-\mathcal{F}_{\mathbf{w}, \mathbf{z}}^{h}(\mathbf{y})\right) .
$$

Let $\left\{\mathbf{x}_{1}, \ldots, \mathbf{x}_{r}\right\} \subset \mathbb{T}_{\alpha} \cap \mathbb{T}_{\beta}$ be the generating set of $\widehat{C F L}\left(Y, L, D_{\text {top }}\right)$. Let

$$
C_{0}=\max _{\mathbf{x} \in \mathbb{T}_{\alpha} \cap \mathbb{T}_{\beta}} \mathcal{F}_{\mathbf{w}, \mathbf{z}}^{h}(\mathbf{x})-D_{\text {top }} \geq 0 .
$$

Lemma 5.14 (Compare [14, Lemma 3.9].) Fix $\mathbf{p}=\left(p_{1}, \ldots, p_{l}\right)$. For all sufficiently large $\mathbf{n}=\left(n_{1}, \ldots, n_{l}\right)$, if $\mathbf{u} \in \mathbb{T}_{\alpha} \cap \mathbb{T}_{\gamma}$ is not an outermost exterior point, then

$$
\mathbf{u} \prec \mathbf{x}_{1}^{\prime} .
$$


Proof Let $-C_{1}$ be a lower bound of $\mathcal{F}_{\mathbf{w}, \mathbf{z}^{\prime}}^{h^{\prime}}\left(\mathbf{x}^{A}\right)-\mathcal{F}_{\mathbf{w}, \mathbf{z}^{\prime}}^{h^{\prime}}(\mathbf{v})$ for all $\mathbf{x} \in \mathbb{T}_{\alpha} \cap \mathbb{T}_{\beta}$, all nonempty $A \subset\{1, \ldots, l\}$, and all type- $A$ interior intersection points $\mathbf{v}$. Lemma 5.9 enables us to choose $C_{1}$ to be a constant independent of $\mathbf{n}$.

Let $\mathbf{n}$ be sufficiently large so that

$$
p_{i} n_{i}>\max \left\{C_{1},\left|p_{i}-2\right| \sum_{j \neq i} p_{j} \cdot\left|\operatorname{lk}\left(K_{i}, K_{j}\right)\right|+m C_{0}\right\}, \quad \text { for } i=1, \ldots, l .
$$

As a result, if $\mathbf{x}^{0}, \mathbf{x}^{s} \in \mathbb{T}_{\alpha} \cap \mathbb{T}_{\gamma}$ differ only at the $\gamma_{i}$-component for some $i$, then Lemma 5.4, Corollary 5.5, (6) and (7) imply that

$$
\mathbf{x}^{s} \prec \mathbf{x}^{0} .
$$

Assume $\mathbf{u}$ is not an outermost exterior point. Let

$A=\left\{i\right.$ : the $\gamma_{i}$-coordinate of $\mathbf{u}$ is not an exterior point $\}$,

$B=\left\{j:\right.$ the $\gamma_{j}$-coordinate of $\mathbf{u}$ is an exterior point but not outermost $\}$.

Then $A \cup B$ is nonempty.

If $A \neq \varnothing$, suppose $i \in A$. For each $j \notin A$, change the $\gamma_{j}$-coordinate of $\mathbf{u}$ to $x_{j}^{0}$, we get a type $-A$ interior point $\overline{\mathbf{u}}$. By Lemma 5.4, Corollary 5.5, (11) and (10) we have

$$
\begin{aligned}
\mathcal{F}_{\mathbf{w}, \mathbf{z}^{\prime}}^{h^{\prime}}\left(\mathbf{x}_{1}^{\prime}\right)-\mathcal{F}_{\mathbf{w}, \mathbf{z}^{\prime}}^{h^{\prime}}(\mathbf{u}) & \geq \mathcal{F}_{\mathbf{w}, \mathbf{z}^{\prime}}^{h^{\prime}}\left(\mathbf{x}_{1}^{\prime}\right)-\mathcal{F}_{\mathbf{w}, \mathbf{z}^{\prime}}^{h^{\prime}}(\overline{\mathbf{u}}) \\
& \geq p_{i} n_{i}+\mathcal{F}_{\mathbf{w}, \mathbf{z}^{\prime}}^{h^{\prime}}\left(\mathbf{x}_{1}^{A}\right)-\mathcal{F}_{\mathbf{w}, \mathbf{z}^{\prime}}^{h^{\prime}}(\overline{\mathbf{u}}) \\
& \geq p_{i} n_{i}-C_{1}>0 .
\end{aligned}
$$

If $A=\varnothing, B \neq \varnothing$, suppose $i \in B$. For any $j$, change the $\gamma_{j}$-coordinate of $\mathbf{u}$ to $x_{j}^{0}$, we get a point $\mathbf{x}^{\prime}$ for some $\mathbf{x} \in \mathbb{T}_{\alpha} \cap \mathbb{T}_{\beta}$. We have

$$
\begin{array}{rlr}
\mathcal{F}_{\mathbf{w}, \mathbf{Z}^{\prime}}^{h^{\prime}}(\mathbf{u}) & \leq \mathcal{F}_{\mathbf{w}, \mathbf{z}^{\prime}}^{h^{\prime}}\left(\mathbf{x}^{\prime}\right)-p_{i} n_{i}+\left|p_{i}-2\right| \sum_{j \neq i} p_{j} \cdot\left|\operatorname{lk}\left(K_{i}, K_{j}\right)\right| & \text { by (7), (11) } \\
& <\mathcal{F}_{\mathbf{w}, \mathbf{Z}^{\prime}}^{h^{\prime}}\left(\mathbf{x}^{\prime}\right)-m C_{0} & \text { by (10) } \\
& \leq \mathcal{F}_{\mathbf{w}, \mathbf{z}^{\prime}}^{h^{\prime}}\left(\mathbf{x}_{1}^{\prime}\right) & \text { by (8), (9). }
\end{array}
$$

Proposition 5.15 With the notation as above, when the winding number $\mathbf{n}$ is sufficiently large,

$$
\widehat{H F L}\left(Y, L, D_{\text {top }}\right) \cong \widehat{H F L}\left(Y, C(L), D_{\text {top }}^{\prime}\right) .
$$

Moreover, suppose $\mathbf{x}$ is one of the generators of $\widehat{C F L}\left(Y, L, D_{\text {top }}\right)$, then $\mathbf{x}^{\prime}$ is one of the generators of $\widehat{C F L}\left(Y, C(L), D_{\text {top }}^{\prime}\right)$. 
Proof Let $\widehat{C F L} \preceq$ be the summand of $\widehat{C F L}(Y, C(L))$, which consists of all the elements with grading no lower than the grading of $\mathbf{x}_{1}^{\prime}$. By Lemma 5.14, the generators of $\widehat{C F L}_{\preceq}$ are all outermost exterior points. The differential on $\widehat{C F L} \preceq$ counts holomorphic disks away from $\mathbf{w}, \mathbf{z}^{\prime}$, denoted by $\partial_{\mathbf{w}, \mathbf{z}^{\prime}}$.

The base points $\mathbf{t}$ give an extra filtration to $\widehat{C F L}_{\preceq}$. If a holomorphic disk $\phi$ connects two exterior points $\overline{\mathbf{y}}_{1}$ to $\overline{\mathbf{y}}_{2}$, and $\phi$ avoids $\mathbf{w}, \mathbf{z}^{\prime}, \mathbf{t}$, then the positivity of $\phi$ implies that the $\gamma_{i}$-components of $\overline{\mathbf{y}}_{1}$ and $\overline{\mathbf{y}}_{2}$ coincide for all $i$. Thus $\phi$ corresponds to a holomorphic disk connecting $\mathbf{y}_{1}$ to $\mathbf{y}_{2}$, which avoids $\mathbf{w}, \mathbf{z}$. Here $\mathbf{y}_{j} \in \mathbb{T}_{\alpha} \cap \mathbb{T}_{\beta}$, $(j=1,2$,) is an intersection point whose components coincide with the components of $\overline{\mathbf{y}}_{j}$, except the $\alpha_{i}$-components. (This correspondence can be seen through the cylindrical reformulation of Heegaard Floer homology due to Lipshitz [7].)

Hence the chain complex $\left(\widehat{C F L}_{\preceq}, \partial_{\mathbf{w}, \mathbf{z}^{\prime}, \mathbf{t}}\right)$ is the direct sum of summands in the form of $\widehat{C F L}_{\mathbf{r}, d}$, where here $\widehat{C F L}_{\mathbf{r}, d}$ is generated by the outermost exterior intersection points $\overline{\mathbf{y}} \in \mathbb{T}_{\alpha} \cap \mathbb{T}_{\gamma}$, which satisfy that the $\gamma_{i}$-component of $\overline{\mathbf{y}}$ is $x_{i}^{r_{i}}$, and the grading difference between $\overline{\mathbf{y}}$ and $\mathbf{x}_{1}^{\prime}$ is $d \geq 0$.

For each generator $\overline{\mathbf{y}}$ of $\widehat{C F L}_{\mathbf{r}, d}$, let $\mathbf{y} \in \mathbb{T}_{\alpha} \cap \mathbb{T}_{\beta}$ be the point whose coordinates are equal to the coordinates of $\overline{\mathbf{y}}$ except the $\alpha_{i}$-coordinates, $i=1, \ldots, l$. By (6) and (8), we have

$$
\begin{aligned}
\mathcal{F}_{\mathbf{w}, \mathbf{z}}^{h}(\mathbf{y})-\mathcal{F}_{\mathbf{w}, \mathbf{z}}^{h}\left(\mathbf{x}_{1}\right) & =\frac{1}{m}\left(\mathcal{F}_{\mathbf{w}, \mathbf{z}^{\prime}}^{h^{\prime}}\left(\mathbf{y}^{\prime}\right)-\mathcal{F}_{\mathbf{w}, \mathbf{z}^{\prime}}^{h^{\prime}}\left(\mathbf{x}_{1}^{\prime}\right)\right) \\
& =\frac{1}{m}\left(\mathcal{F}_{\mathbf{w}, \mathbf{z}^{\prime}}^{h^{\prime}}\left(\mathbf{y}^{\prime}\right)-\mathcal{F}_{\mathbf{w}, \mathbf{z}^{\prime}}^{h^{\prime}}(\overline{\mathbf{y}})+d\right) \\
& =\frac{1}{m}\left(\sum_{i=1}^{l}\left(S_{i}\left(x_{i}^{0}\right)-S_{i}\left(x_{i}^{r_{i}}\right)\right)+d\right) \geq 0 .
\end{aligned}
$$

So the homology of $\left(\widehat{C F L}_{\mathbf{r}, d}, \partial_{\mathbf{w}, \mathbf{z}^{\prime}, \mathbf{t}}\right)$ is isomorphic to the homology of some summand of $\widehat{C F L}(Y, L)$, at a fixed grading no less than the grading of $\mathbf{x}_{1}$.

Since $\mathbf{x}_{1}$ lies in the topmost nontrivial Alexander $\mathbb{Q}$-grading of $\widehat{H F L}(Y, L)$, we find that $\widehat{H F L}_{\mathbf{r}, d}$ is nontrivial if and only if $(\mathbf{r}, d)=(\mathbf{0}, 0)$, and

$$
\widehat{H F L}_{\mathbf{0}, 0} \cong \widehat{H F L}\left(Y, L, D_{\text {top }}\right) \text {. }
$$

There is a spectral sequence which starts from $\left(\widehat{C F L}_{\preceq}, \partial_{\mathbf{w}, \mathbf{z}^{\prime}, \mathbf{t}}\right)$, and converges to $H\left(\widehat{C F L}_{\preceq}, \partial_{\mathbf{w}, \mathbf{z}^{\prime}}\right)$. Since the $E^{2}$ term is only supported in one filtration level,

$$
H\left(\widehat{C F L}_{\preceq}, \partial_{\mathbf{w}, \mathbf{z}^{\prime}}\right) \cong \widehat{H F L}_{\mathbf{0}, 0}
$$

Thus

$$
\widehat{H F L}\left(Y, C(L), D_{\text {top }}^{\prime}\right) \cong \widehat{H F L}\left(Y, L, D_{\text {top }}\right) \text {. }
$$

The last statement of this proposition is obvious from the proof. 
Our next task is to determine the absolute position of the topmost grading in $\widehat{H F L}(Y, L)$. For this purpose, we will consider two relative $\operatorname{Spin}^{c}$ structures $\mathfrak{r}_{1}, \mathfrak{r}_{2} \in \operatorname{Spin}^{c}(Y, C(L))$. Given $\mathbf{x} \in \mathbb{T}_{\alpha} \cap \mathbb{T}_{\beta}$, let $\mathfrak{r}_{1}=\underline{\mathfrak{s}}_{\mathbf{w}, \mathbf{z}^{\prime}}\left(\mathbf{x}^{\prime}\right) \in \underline{\operatorname{Spin}}^{c}(Y, C(L))$.

Construction 5.16 A vector field $v_{1}$ representing $\mathfrak{r}_{1}$ can be constructed as in Section 4.2. Let $f: Y \rightarrow[0,3]$ be a Morse function corresponding to the Heegaard diagram $(\Sigma, \boldsymbol{\alpha}, \boldsymbol{\gamma})$. There is a nowhere vanishing vector field $\widetilde{v}_{1}$ on $Y$ as follows. Outside a neighborhood of $\gamma_{\mathbf{w}} \cup \gamma_{\mathbf{z}^{\prime}} \cup \gamma_{\mathbf{x}^{\prime}}, \widetilde{v}_{1}$ is identical with $\nabla f$. We then extend the vector field over the balls $\operatorname{Nd}\left(\gamma_{\mathbf{x}^{\prime}}\right)$, and over $\operatorname{Nd}\left(\gamma_{\mathbf{w}} \cup \gamma_{\mathbf{z}^{\prime}}\right)$ as in Section 4.2. The closed orbits of $\widetilde{v}_{1}$ which pass through points in $\mathbf{w}, \mathbf{z}^{\prime}$ are identical with the oriented link $C(L)=\gamma_{\mathbf{z}^{\prime}}-\gamma_{\mathbf{w}}$. The vector field $v_{1}$ is the restriction of $\tilde{v}_{1}$ to $Y-\operatorname{Nd}(C(L))$.

The cable space $\operatorname{Nd}\left(K_{i}\right)-\operatorname{int}\left(\operatorname{Nd}\left(C_{p_{i}, q_{i}}\left(K_{i}\right)\right)\right)$ fibers over the circle, with fiber $G_{i}$. Let $u_{i}$ be a vector field on the cable space, which is transverse to the fibers everywhere, and the orientation of $u_{i}$ is opposite to the orientation induced by the orientation of the fibers. Under this assumption, the restriction of $u_{i}$ on the boundary tori is isotopic to a translation invariant vector field, which is unique up to isotopy.

Note that $\underline{\mathfrak{s}}_{\mathbf{w}, \mathbf{z}}(\mathbf{x})=\mathfrak{\mathfrak { s }}_{\mathbf{w}, \mathbf{t}}\left(\mathbf{x}^{\prime}\right)$ is a relative $\operatorname{Spin}^{c}$ structure on $Y-\operatorname{int}(\operatorname{Nd}(L))$, we can extend it to a relative $\operatorname{Spin}^{c}$ structure on $Y-\operatorname{int}(\operatorname{Nd}(C(L)))$ by the vector fields $u_{1}, \ldots, u_{l}$. We denote this new $\operatorname{Spin}^{c}$ structure by $\mathfrak{r}_{2}$.

Construction 5.17 A vector field $v_{2}$ representing $\mathfrak{r}_{2}$ can be constructed as follows. First construct a nowhere vanishing vector field $\widetilde{v}_{2}$ on $Y$. Outside a neighborhood of $\gamma_{\mathbf{w}} \cup \gamma_{\mathbf{t}} \cup \gamma_{\mathbf{x}^{\prime}}, \tilde{v}_{2}$ is identical with $\nabla f$. We then extend the vector field over the balls $\operatorname{Nd}\left(\gamma_{\mathbf{x}^{\prime}}\right)$, and over $\operatorname{Nd}\left(\gamma_{\mathbf{w}} \cup \gamma_{\mathbf{t}}\right)$ as in Section 4.2. The closed orbits of $\widetilde{v}_{2}$ which pass through points in $\mathbf{w}, \mathbf{t}$ are identical with the oriented link $L=\gamma_{\mathbf{t}}-\gamma_{\mathbf{w}}$. The vector field $v_{2}$ is the union of $\left.\tilde{v}_{2}\right|_{Y-\operatorname{Nd}(L)}$ and $u_{1}, \ldots, u_{l}$. (We should isotope $u_{i}$ 's near the boundary of the cable spaces so that they are identical to $\left.\tilde{v}_{2}\right|_{Y-\mathrm{Nd}(L)}$ on $\partial \mathrm{Nd}(L)$.)

We define two functions

$$
\mathcal{F}_{1}, \mathcal{F}_{2}: \mathbb{T}_{\alpha} \cap \mathbb{T}_{\beta} \rightarrow \mathbb{Q}
$$

The function $\mathcal{F}_{1}$ is defined as follows:

$$
\mathcal{F}_{1}(\mathbf{x})=\left\langle\mathfrak{H}_{C(L)}\left(\mathfrak{r}_{1}\right), h^{\prime}\right\rangle,
$$

where here $\mathfrak{H}_{C(L)}$ is the affine map defined in Section 4.4, for the pair $(Y, C(L))$. More precisely,

$$
\mathcal{F}_{1}(\mathbf{x})=\left\langle\frac{c_{1}\left(\mathfrak{r}_{1}\right)-\sum_{i=1}^{l} \operatorname{PD}\left(\left[\mu_{i}^{\prime}\right]\right)}{2}, h^{\prime}\right\rangle .
$$


The function $\mathcal{F}_{2}$ is defined as

$$
\mathcal{F}_{2}(\mathbf{x})=\left\langle\frac{c_{1}\left(\mathfrak{r}_{2}\right)-\sum_{i=1}^{l} \operatorname{PD}\left(\iota_{*}\left[\mu_{i}\right]\right)}{2}, h^{\prime}\right\rangle .
$$

Let $\rho:(Y, C(L)) \rightarrow(Y, \operatorname{Nd}(L))$ be the inclusion map of pairs, then $\rho_{*}\left(h^{\prime}\right)=m \cdot h$. By the choice of $u_{i}$, the evaluation of $c_{1}\left(u_{i}\right)$ on $G_{i}$ is always $-\chi\left(G_{i}\right)$, where $c_{1}\left(u_{i}\right)$ is the Chern class of the relative $\operatorname{Spin}^{c}$ structure determined by $u_{i}$. So

$$
\begin{aligned}
\mathcal{F}_{2}(\mathbf{x}) & \left.=\left\langle\frac{c_{1}\left(\underline{\mathfrak{g}}_{\mathbf{w}, \mathbf{z}}(\mathbf{x})\right)-\sum_{i=1}^{l} \operatorname{PD}\left(\left[\mu_{i}\right]\right)}{2}, \rho_{*}\left(h^{\prime}\right)\right\rangle+\frac{1}{2} \sum_{i=1}^{l}\left\langle c_{1}\left(u_{i}\right),\left[G_{i}\right]\right\rangle\right\rangle \\
& =m\left\langle\mathfrak{H}\left(\underline{\mathfrak{g}}_{\mathbf{w}, \mathbf{z}}(\mathbf{x})\right), h\right\rangle-\frac{1}{2} \sum_{i=1}^{l} \chi\left(G_{i}\right) .
\end{aligned}
$$

In summary, $\mathcal{F}_{1}$ and $\mathcal{F}_{2}$ can be factorized as follows:

$$
\begin{aligned}
& \mathcal{F}_{1}: \mathbb{T}_{\alpha} \cap \mathbb{T}_{\beta} \rightarrow \mathbb{T}_{\alpha} \cap \mathbb{T}_{\gamma} \rightarrow \underline{\operatorname{Spin}^{c}(Y, C(L)) \rightarrow \mathbb{Q},} \\
& \mathcal{F}_{2}: \mathbb{T}_{\alpha} \cap \mathbb{T}_{\beta} \rightarrow \underline{\operatorname{Spin}^{c}}(Y, L) \rightarrow \underline{\operatorname{Spin}^{c}}(Y, C(L)) \rightarrow \mathbb{Q} .
\end{aligned}
$$

Let max $\mathcal{F}_{1}$ be the value of $\mathcal{F}_{1}(\mathbf{x})$ for intersection point $\mathbf{x}$ such that the grading of $\mathbf{x}^{\prime}$ is $D_{\text {top }}^{\prime}$. Let $\max \mathcal{F}_{2}$ be the value of $\mathcal{F}_{2}(\mathbf{x})$ such that the grading of $\mathbf{x}$ is $D_{\text {top }}$.

From Lemma 5.10, we can conclude that

$$
\mathcal{F}_{1}(\mathbf{x})-\mathcal{F}_{1}(\mathbf{y})=\mathcal{F}_{2}(\mathbf{x})-\mathcal{F}_{2}(\mathbf{y}) .
$$

In fact, we can prove something stronger:

Proposition 5.18 Given $\mathbf{x} \in \mathbb{T}_{\alpha} \cap \mathbb{T}_{\beta}$, then the following equality holds:

$$
\mathcal{F}_{2}(\mathbf{x})=\mathcal{F}_{1}(\mathbf{x})+\sum_{i=1}^{l} \frac{p_{i}-1}{2} .
$$

Proof We could prove (14) by examining the relative $\operatorname{Spin}^{c}$ structures carefully, but we would rather argue via a model computation.

By Constructions 5.16 and 5.17, the two vector fields $v_{1}, v_{2}$ are equal outside a regular neighborhood of the flowlines $\gamma_{\mathbf{w}}, \gamma_{\mathbf{z}^{\prime}}, \gamma_{\mathbf{t}}$. So the difference of $v_{1}$ and $v_{2}$ only depends on their difference inside $\operatorname{Nd}\left(\gamma_{\mathbf{w}} \cup \gamma_{\mathbf{z}^{\prime}} \cup \gamma_{\mathbf{t}}\right)$, which only depends only on the $2 l$ torus link types $\left(p_{i}, q_{i}\right),\left(m P_{i}, m Q_{i}\right), i=1, \ldots, l$. In order to compute the difference of their evaluations (of the Chern classes of the two $\operatorname{Spin}^{c}$ structures) on $F^{\prime}$, we only need to compute the difference of their (relative) evaluations on $F^{\prime} \cap \operatorname{Nd}\left(\gamma_{\mathbf{w}} \cup \gamma_{\mathbf{z}^{\prime}} \cup \gamma_{\mathbf{t}}\right)$. 
So we only need to verify (14) for some model, in which the local behavior of $v_{1}, v_{2}$ are the same as in the general case.

Let $d_{i}=\operatorname{gcd}\left(P_{i}, Q_{i}\right), P_{i}=d_{i} P_{i}^{\prime}, Q_{i}=d_{i} Q_{i}^{\prime}$. The model we are considering is the knot $O_{P_{i}^{\prime}} / Q_{i}^{\prime}$ in $L\left(P_{i}^{\prime}, Q_{i}^{\prime}\right)$. There is an essential disk $D$ properly embedded in the complement of $K=O_{P_{i}^{\prime}} / Q_{i}^{\prime} . \partial D$ is the torus knot $T\left(P_{i}^{\prime}, Q_{i}^{\prime}\right)$ in $\partial \mathrm{Nd}(K)$. Let $F_{0}$ be the union of $d_{i}$ copies of $D$.

Fix a frame on $K$, let $C(K)$ be the $\left(p_{i}, q_{i}\right)$-cable of $K$ with respect to this frame. Let $G_{0} \subset \mathrm{Nd}(K)-\operatorname{int}(\mathrm{Nd}(C(K)))$ be a surface such that $\partial G_{0}$ consists of the torus link $T\left(m P_{i}, m Q_{i}\right)$ and a longitude of $C(K)$, and $\operatorname{Nd}(K)-\operatorname{int}(\operatorname{Nd}(C(K)))$ fibers over the circle with fiber $G_{0} . F_{0}^{\prime}$ is the union of $G_{0}$ and $m$ parallel copies of $F_{0}$.

Now $F_{0}, F_{0}^{\prime}, G_{0}$ play the roles of $F, F^{\prime}, G_{i}$.

From Lemma 5.1, we know that for the pair $\left(L\left(P_{i}^{\prime}, Q_{i}^{\prime}\right), O_{P_{i}^{\prime}} / Q_{i}^{\prime}\right)$

$$
\begin{aligned}
\max \mathcal{F}_{2} & =\frac{1}{2}\left(m d_{i}\left(2 P_{i}^{\prime}-1\right)-\chi\left(G_{0}\right)-p_{i}\right) \\
& =\frac{1}{2}\left(p_{i}-m d_{i}-\chi\left(G_{0}\right)\right)
\end{aligned}
$$

The knot $C(K)$ is null-homologous, so we can apply Proposition 5.2 and Proposition 5.15 to show that

$$
\begin{aligned}
\max \mathcal{F}_{1} & =\frac{1-\chi\left(F_{0}^{\prime}\right)}{2} \\
& =\frac{1}{2}\left(1-m d_{i}-\chi\left(G_{0}\right)\right)
\end{aligned}
$$

So we get

$$
\mathcal{F}_{2}(\mathbf{x})-\mathcal{F}_{1}(\mathbf{x})=\frac{p_{i}-1}{2}
$$

This finishes the model computation, hence (14) holds in general.

Proof of Theorem 1.1 We first prove the case where $\partial M$ is incompressible, thus we only need to prove the statement in Remark 1.2. The homogeneity of Thurston norm enables us to consider the problem for the more general case where $h \in H_{2}(Y, L ; \mathbb{Q})$ is a rational class. By the continuity of Thurston norm, it suffices to prove the theorem for the rational classes $h$ with $h \cdot\left[\mu_{i}\right] \neq 0$ for all $i$. Again by the homogeneity of Thurston norm, we only need to consider the case where $h$ is an integral classes. After changing the orientations of some components, we may assume $h \cdot\left[\mu_{i}\right]>0$ for all $i$.

With the notation as before, consider the $(\mathbf{p}, \mathbf{q})$-cable $C(L)$ of $L$. Here we choose $m=$ 1 , so $p_{i}=P_{i}$. Let $\mathbf{n}=\left(n_{1}, \ldots, n_{l}\right)$ be sufficiently large. Since $\partial M$ is incompressible, 
there exists a surface $F$ representing $h$, and $|\chi(F)|=\chi(h)$. Construct the surfaces $G_{i}, F^{\prime}$ as in Construction 5.12.

We have

$$
\begin{aligned}
\max \{\langle\mathfrak{H}(\mathfrak{r}), h\rangle \mid \widehat{H F L}(Y, L, \mathfrak{r}) \neq 0\} \\
=\max \mathcal{F}_{2}+\frac{1}{2} \sum_{i=1}^{l} \chi\left(G_{i}\right) \\
=\max \mathcal{F}_{1}+\sum_{i=1}^{l} \frac{p_{i}-1}{2}+\frac{1}{2} \sum_{i=1}^{l} \chi\left(G_{i}\right) \\
=\frac{1}{2}\left(l-\chi\left(F^{\prime}\right)\right)+\frac{1}{2} \sum_{i=1}^{l}\left(p_{i}-1\right)+\frac{1}{2} \sum_{i=1}^{l} \chi\left(G_{i}\right) \\
=\frac{1}{2}\left(\sum_{i=1}^{l}\left|[F] \cdot\left[\mu_{i}\right]\right|-\chi(F)\right) .
\end{aligned}
$$

This finishes the proof in the case when $\partial M$ is incompressible.

If $\partial M$ is compressible, say, $\partial \mathrm{Nd}\left(K_{1}\right)$ is compressible. We can compress this boundary torus to get a separating sphere, which splits off a lens space summand from $Y$, and $K_{1}$ is a knot $O_{p / q}$ in this summand. Let $L^{\prime}=L-K_{1}, Y=Y^{\prime} \# L(p, q)$. If $h \in H_{2}(Y, L)$ is an integral class, and $F \subset Y-\operatorname{int}(\operatorname{Nd}(L))$ realizes $\chi(h)$, then $F$ is the disjoint union of some disks in $L(p, q)-\operatorname{int}\left(\operatorname{Nd}\left(O_{p / q}\right)\right)$ and a surface $F^{\prime} \subset Y^{\prime}-\operatorname{int}\left(\operatorname{Nd}\left(L^{\prime}\right)\right)$. We can make use of Proposition 4.3 and Lemma 5.1 to reduce our problem to $L^{\prime}$. Now the proof of our theorem can be finished by induction on $|L|$.

\section{References}

[1] D Gabai, private communication

[2] D Gabai, Foliations and the topology of 3-manifolds, J. Differential Geom. 18 (1983) 445-503 MR723813

[3] D Gabai, Foliations and the topology of 3-manifolds. III, J. Differential Geom. 26 (1987) 479-536 MR910018

[4] M Hedden, On knot Floer homology and cabling, Algebr. Geom. Topol. 5 (2005) 1197-1222 MR2171808

[5] U Kaiser, Link theory in manifolds, Lecture Notes in Math. 1669, Springer, Berlin (1997) MR1479639 
[6] E Kalfagianni, Power series link invariants and the Thurston norm, Topology Appl. 101 (2000) 107-119 MR1732063

[7] R Lipshitz, A cylindrical reformulation of Heegaard Floer homology, Geom. Topol. 10 (2006) 955-1097 MR2240908

[8] Y Ni, A note on knot Floer homology of links, Geom. Topol. 10 (2006) 695-713 MR2240902

[9] P Ozsváth, Z Szabó, Knot Floer homology and rational surgeries arXiv: math.GT/0504404

[10] P Ozsváth, Z Szabó, Holomorphic disks and genus bounds, Geom. Topol. 8 (2004) 311-334 MR2023281

[11] P Ozsváth, Z Szabó, Holomorphic disks and knot invariants, Adv. Math. 186 (2004) 58-116 MR2065507

[12] P Ozsváth, Z Szabó, Holomorphic triangle invariants and the topology of symplectic four-manifolds, Duke Math. J. 121 (2004) 1-34 MR2031164

[13] P Ozsváth, Z Szabó, Holomorphic disks, link invariants and the multi-variable Alexander polynomial, Algebr. Geom. Topol. 8 (2008) 615-692 MR2443092

[14] P Ozsváth, Z Szabó, Link Floer homology and the Thurston norm, J. Amer. Math. Soc. 21 (2008) 671-709 MR2393424

[15] J Rasmussen, Floer homology and knot complements, $\mathrm{PhD}$ thesis, Harvard University (2003) arXiv:math.GT/0306378

[16] D Rolfsen, Knots and links, Math. Lecture Ser. 7, Publish or Perish, Berkeley, CA (1976) MR0515288

[17] M Scharlemann, A Thompson, Link genus and the Conway moves, Comment. Math. Helv. 64 (1989) 527-535 MR1022995

[18] F Sergeraert, Feuilletages et difféomorphismes infiniment tangents à l'identité, Invent. Math. 39 (1977) 253-275 MR0474327

[19] W P Thurston, A norm for the homology of 3-manifolds, Mem. Amer. Math. Soc. 59 (1986) i-vi and 99-130 MR823443

Department of Mathematics, Columbia University MC 4406, 2990 Broadway, New York, NY 10027

yni@math. columbia.edu

Proposed: Peter Ozsváth

Received: 8 May 2006

Seconded: Ron Fintushel, Walter Neumann

Revised: 2 July 2009 